\title{
EXTENDING GEOMETRIC SINGULAR PERTURBATION THEORY TO NONHYPERBOLIC POINTS-FOLD AND CANARD POINTS IN TWO DIMENSIONS*
}

\author{
M. KRUPA ${ }^{\dagger}$ AND P. SZMOLYAN ${ }^{\dagger}$
}

\begin{abstract}
The geometric approach to singular perturbation problems is based on powerful methods from dynamical systems theory. These techniques have been very successful in the case of normally hyperbolic critical manifolds. However, at points where normal hyperbolicity fails, the well-developed geometric theory does not apply. We present a method based on blow-up techniques, which leads to a rigorous geometric analysis of these problems. A detailed analysis of the extension of slow manifolds past fold points and canard points in planar systems is given. The efficient use of various charts is emphasized.
\end{abstract}

AMS subject classifications. 34E15, 34E05, 34E20, 34C20, 34C26, 34C $30,34 \mathrm{C} 40,37 \mathrm{C} 10$

Key words. singular perturbations, slow manifolds, nonhyperbolicity, blow-up, folds, canards

PII. S0036141099360919

1. Introduction. We consider singularly perturbed ordinary differential equations (ODEs) in the standard form

$$
\begin{aligned}
\varepsilon \dot{x} & =f(x, y, \varepsilon), \quad x \in \mathbb{R}^{n}, \quad y \in \mathbb{R}^{m}, \quad 0<\varepsilon \ll 1, \\
\dot{y} & =g(x, y, \varepsilon), \quad
\end{aligned}
$$

where $f, g$ are $C^{k}$-functions with $k \geq 3$. Properties of solutions of (1.1) can be studied using geometric methods from dynamical systems theory. This approach, known as geometric singular perturbation theory, has been very successful in many contexts, yet has encountered difficulties in certain situations. In this article we show how some of the limitations of geometric singular perturbation theory can be removed.

Before describing our results we present a brief survey of the existing theory. Let $\tau$ denote the independent variable in (1.1). The variable $\tau$ is referred to as the slow time scale. By switching to the fast time scale $t:=\tau / \varepsilon$ one obtains the equivalent system

$$
\begin{aligned}
& x^{\prime}=f(x, y, \varepsilon), \\
& y^{\prime}=\varepsilon g(x, y, \varepsilon) .
\end{aligned}
$$

One tries to analyze the dynamics of (1.1) by suitably combining the dynamics of the reduced problem

$$
\begin{aligned}
& 0=f(x, y, 0), \\
& \dot{y}=g(x, y, 0)
\end{aligned}
$$

${ }^{*}$ Received by the editors September 1, 1999; accepted for publication (in revised form) December 16, 2000; published electronically June 8, 2001.

http://www.siam.org/journals/sima/33-2/36091.html

${ }^{\dagger}$ Institut für Angewandte und Numerische Mathematik, Technische Universität Wien, Wiedner Hauptstraße 6-10, A-1040 Wien, Austria (peter.szmolyan@tuwien.ac.at). This research was supported by the Austrian Science Foundation under grant Y 42-MAT.

${ }^{\ddagger}$ Current address: Department of Mathematical Sciences, New Mexico State University, Las Cruces, NM 88003-8001 (mkrupa@nmsu.edu). 
and the dynamics of the layer problem

$$
\begin{aligned}
& x^{\prime}=f(x, y, 0), \\
& y^{\prime}=0,
\end{aligned}
$$

which are the limiting problems for $\varepsilon=0$ on the slow and the fast time scales, respectively.

The foundation of geometric singular perturbation theory was laid by Fenichel [8]. The basic reasoning is as follows. The reduced problem (1.3) is a dynamical system on the set $S:=\left\{(x, y) \in \mathbb{R}^{n+m}: f(x, y, 0)=0\right\}$. In the following we refer to $S$ as the critical manifold. A normally hyperbolic invariant manifold of equilibria $S_{0} \subset S$ of the layer problem (1.4) persists as a locally invariant slow manifold $S_{\varepsilon}$ of (1.1) for $\varepsilon$ sufficiently small. The restriction of (1.1) to $S_{\varepsilon}$ is a small smooth perturbation of the reduced problem (1.3). Moreover, there exist a stable and an unstable invariant foliation with base $S_{\varepsilon}$ with the dynamics along each foliation being a small perturbation of the suitable restriction of the dynamics of (1.4). For an excellent introduction to geometric singular perturbation theory and an overview of applications, we refer the reader to the survey by Jones [11].

However, despite many efforts, points on the critical manifold $S$ where normal hyperbolicity breaks down remained a major obstacle to the geometric theory. This was a definite shortcoming in view of the abundance of nonhyperbolic points in applications.

One cause for the breakdown of normal hyperbolicity of a critical manifold $S$ are bifurcation points due to a zero eigenvalue of the Jacobian $\frac{\partial f}{\partial x}$. The most common case are folded critical manifolds. A well-known phenomenon in this context are relaxation oscillations, i.e., solutions slowly moving towards a fold point, jumping from the fold point to another stable branch of $S$, following the slow dynamics again until another fold point is reached, jumping again, etc., thus, possibly forming periodic solutions [9], [18], and [20].

Another delicate phenomenon occuring at folds are canard solutions which were discovered and first analyzed by Benoit, Callot, Diener, and Diener [3]; see also [2]. A canard solution is a solution of a singularly perturbed system which is contained in the intersection of an attracting slow manifold and a repelling slow manifold. The existence of a canard solution can lead to canard explosion, i.e., a transition from a small limit cycle to a relaxation oscillation through a sequence of canard cycles [3], [6], [7]. For planar vector fields canards are nongeneric and occur persistently in one-parameter families; yet in dimensions larger than two they can occur in generic situations [2], [19], [23].

In this article we show how geometric singular perturbation theory can be extended to fold points and canard points in planar systems, i.e., we restrict our attention to the case $n=m=1$. A fold point corresponds to the situation when the critical manifold has a generic fold. Depending on the stability properties of the critical manifold and on the direction of the reduced flow, a number of cases are possible. We analyze the so-called jump point, for which the reduced flow is directed towards the fold. This is the situation which is relevant for relaxation oscillations. We show how the slow manifolds (existing by the normally hyperbolic theory) extend in the neighborhood of the singularity. The treatment of the fold point is a refinement of the analysis in our earlier work [13]. A canard point is a fold point with an additional degeneracy leading to a possibility of a canard solution. Again we analyze how slow manifolds extend and show that a canard solution occurs along a codimension one 
curve in the parameter plane. In a complementary work [15] we carry out a similar analysis for singularities of pitchfork and transcritical type.

Our approach relies on the blow-up method, which is a way of partially desingularizing the vector field in the neighborhood of a singular point. After a blow-up transformation, standard methods from dynamical systems theory can be applied. Our proof of the existence of a canard solution is based on a variant of the Melnikov method. The blow-up method was first applied to a singular perturbation problem in the pioneering work of Dumortier and Roussarie [6], who analyzed the existence of canard cycles in the van der Pol equation. One of the purposes of this work is to build a bridge between the methods of [6] and geometric singular perturbation theory; in particular, we use the blow-up method to answer the question of extending slow manifolds near nonhyperbolic singularities.

The sequel of this article [14] is devoted to relaxation oscillations and canard explosion. The methods and results of this paper are of central importance in our analysis of these phenomena. Since the analysis of canard cycles is a more delicate problem, we find it advantageous to treat these issues separately. In particular, we prefer to use blow-up to obtain local results which in a second step can be used to study global phenomena. We feel that this point of view is also useful in the analysis of related problems. In this sense, our work is intended as a complement to the more global approach in the work of Dumortier [5] and Dumortier and Roussarie [6].

In this article and in [14] we restrict our attention to the planar case. However, the analysis carries over to higher-dimensional problems with one-dimensional critical manifolds containing fold points. By means of a center-manifold reduction, all normally hyperbolic directions can be eliminated and one recovers the planar problems considered here. A well-known problem where this is relevant is the traveling wave problem for the FitzHugh-Nagumo equation [11]. For a similar approach to problems with higher-dimensional critical manifolds, we refer the reader to [17] and [23].

The article is organized as follows. Section 2 contains the description and the analysis of a generic fold. Here we give a detailed expository presentation of the blow-up method. In section 3 we analyze a canard point.

\section{Generic fold.}

2.1. Assumptions and results. Consider the singularly perturbed ODE (1.2), where $(x, y) \in \mathbb{R}^{2}$ and $\varepsilon$ is a small real parameter. Suppose that $\left(x_{0}, y_{0}\right)$ is such that

$$
f\left(x_{0}, y_{0}, 0\right)=0, \quad \frac{\partial f}{\partial x}\left(x_{0}, y_{0}, 0\right)=0 .
$$

Our goal is to obtain a characterization of the dynamics in a neighborhood of $\left(x_{0}, y_{0}\right)$ for sufficiently small values of $\varepsilon$. We make the following nondegeneracy assumptions:

$$
\frac{\partial^{2} f}{\partial x^{2}}\left(x_{0}, y_{0}, 0\right) \neq 0, \quad \frac{\partial f}{\partial y}\left(x_{0}, y_{0}, 0\right) \neq 0, \quad g\left(x_{0}, y_{0}, 0\right) \neq 0 .
$$

We assume, without loss of generality, that

$$
\left(x_{0}, y_{0}\right)=(0,0), \quad \frac{\partial^{2} f}{\partial x^{2}}(0,0,0)>0, \quad \frac{\partial f}{\partial y}\left(x_{0}, y_{0}, 0\right)<0
$$

hold.

As before let $S=\{(x, y): f(x, y, 0)=0\}$ be the critical manifold. The nondegeneracy assumptions imply that there exists a neighborhood $U$ of the origin such that 


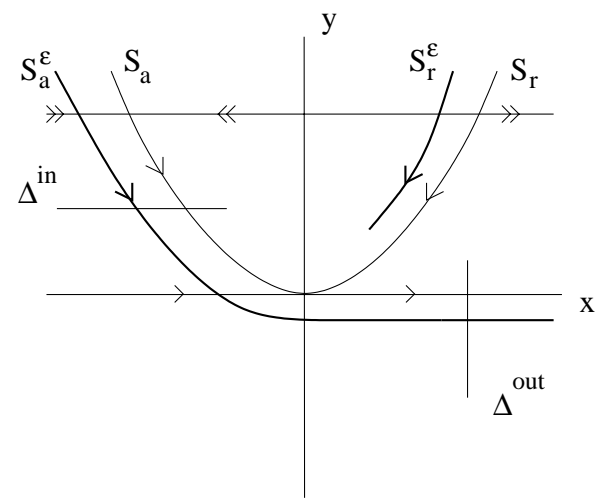

FIG. 2.1. Critical manifold, slow manifolds, and sections for the fold point.

$(0,0)$ is the only point in $U \cap S$, where $\frac{\partial f}{\partial x}$ vanishes and that $S \cap U$ is approximately a parabola. Let $S_{a}$ (resp., $S_{r}$ ) denote its left (resp., right) branch, so that $S=S_{a} \cup S_{r}$ (see Figure 2.1). The assumption $\frac{\partial^{2} f}{\partial x^{2}}(0,0,0)>0$ implies that for $y>0$ the branch $S_{a}$ is attracting and the branch $S_{r}$ is repelling for the layer problem, which also explains the notation. The origin is nonhyperbolic, weakly attracting from the left and weakly repelling to the right (see Figure 2.1).

To determine the reduced dynamics we solve the equation $f(x, y, 0)=0$ for $y$ as a function of $x$, i.e., $y=\varphi(x)$. The reduced dynamics is then determined by

$$
\varphi^{\prime}(x) \dot{x}=g(x, \varphi(x), 0),
$$

which is singular at $x=0$. Our assumptions on $f$ and $g$ imply that the direction of the reduced flow is determined by the sign of $g(0,0,0)$. We assume $g(0,0,0)<0$. This implies that the reduced flow on $S_{a}$ and $S_{r}$ is directed towards the fold point; see Figure 2.1. Actually, orbits on $S_{a}$ and $S_{r}$ reach the fold point in finite time due to the singularity at the fold point. The only possibility to continue from there in the singular limit is along the (weakly) unstable fiber of the layer problem along the positive $x$-axis. Thus, the curve $S_{a} \cup\{(x, 0), x>0\}$ is expected to be a zeroth order approximation. This is the situation relevant to relaxation oscillations; we refer to this case as jump point. The case $g(0,0,0)>0$ can be analyzed similarly.

It follows from the standard theory [8] that outside an arbitrarily small neighborhood $V$ of $(0,0)$, the manifolds $S_{a}$ and $S_{r}$ perturb smoothly to locally invariant manifolds $S_{a, \varepsilon}$ and $S_{r, \varepsilon}$ for sufficiently small $\varepsilon \neq 0$. We would like to point out that $S_{a, \varepsilon}$ and $S_{r, \varepsilon}$ are actually very simple. They consist of single solutions. Note that the slow manifolds are obtained as sections $\varepsilon=$ const. of two-dimensional, locally invariant, center-like manifolds $M_{a}$ (resp., $M_{r}$ ) of the extended system

$$
\begin{aligned}
& x^{\prime}=f(x, y, \varepsilon), \\
& y^{\prime}=\varepsilon g(x, y, \varepsilon), \\
& \varepsilon^{\prime}=0
\end{aligned}
$$

in the extended phase space $\mathbb{R}^{3}$. For this extended system $S \times\{0\}$ is a manifold of equilibria. Outside of a neighborhood of the fold point $(0,0,0)$ the linearization of system (2.4) at points $S_{a} \times\{0\}$ has a double zero eigenvalue and one uniformly hyperbolic (stable) eigenvalue. This allows us to conclude the existence of the attracting 
center-like manifold $M_{a}$; the manifold $M_{r}$ is obtained in a similar way. At the fold point $(0,0,0)$ the linearization has a triple eigenvalue zero and the construction of the slow manifolds breaks down. We focus our attention on $S_{a}$ and investigate how $S_{a, \varepsilon}$ as well as nearby solutions behave as they pass near the fold point. We expect that close to the fold point a transition from slow motion along $S_{a, \varepsilon}$ to a fast motion almost parallel to the unstable fibers occurs. A similar analysis could be carried out for $S_{r, \varepsilon}$.

Remark 2.1. It is known that the slow manifolds $M_{a}$ and $M_{r}$ and hence their sections $S_{a, \varepsilon}$ and $S_{r, \varepsilon}$ are not unique and are determined only up to $O\left(e^{-c / \varepsilon}\right)$, where $c$ is some positive constant. We make an arbitrary choice of $M_{a}$ and $M_{r}$ and indicate at the end that our results are independent of this choice.

We now view the previously introduced neighborhood $U$ as a neighborhood of $(0,0,0)$ in $\mathbb{R}^{3}$. We pick $U$ sufficiently small, so that $g(x, y, \varepsilon) \neq 0$ for $(x, y, \varepsilon) \in U$. Before stating the main results we rewrite system (1.2) (resp., (2.4)) in a canonical form. By rescaling $x, y, \varepsilon$, and $t$ we obtain

$$
\begin{aligned}
& x^{\prime}=-y+x^{2}+h(x, y, \varepsilon), \\
& y^{\prime}=\varepsilon g(x, y, \varepsilon), \\
& \varepsilon^{\prime}=0
\end{aligned}
$$

with $h(x, y, \varepsilon)=O\left(\varepsilon, x y, y^{2}, x^{3}\right), g(x, y, \varepsilon)=-1+O(x, y, \varepsilon)$, where the new function $g$ is related to the original one by the rescaling. This form of the equations will be used throughout the forthcoming analysis.

For small $\rho>0$ and a suitable interval $J \subset \mathbb{R}$ let

$$
\Delta^{i n}=\left\{\left(x, \rho^{2}\right), x \in J\right\}
$$

be a section in $U$ transverse to $S_{a}$ and let

$$
\Delta^{\text {out }}=\{(\rho, y), y \in \mathbb{R}\}
$$

be a section in $U$ transverse to the fast fibers (see Figure 2.1). Note, that the same constant $\rho$ is used throughout this paper.

Let $\pi: \Delta^{\text {in }} \rightarrow \Delta^{\text {out }}$ be the transition map for the flow of (1.2).

THEOREM 2.1. Under the assumptions made in this section there exists $\varepsilon_{0}>0$ such that the following assertions hold for $\varepsilon \in\left(0, \varepsilon_{0}\right]$ :

1. The manifold $S_{a, \varepsilon}$ passes through $\Delta^{\text {out }}$ at a point $(\rho, h(\varepsilon))$, where $h(\varepsilon)=$ $O\left(\varepsilon^{2 / 3}\right)$.

2. The transition map $\pi$ is a contraction with contraction rate $O\left(e^{-c / \varepsilon}\right)$, where $c$ is a positive constant.

In the context of matched asymptotic expansions assertion (1) of the theorem is well known; see, e.g., [18]. A blow-up based derivation of the asymptotic expansion of $h(\varepsilon)$ is given in [16]. Assertion (2) of the theorem explains why the nonuniqueness of the slow manifold $M_{a}$ (resp., $S_{a, \varepsilon}$ ) does not affect our results. Two different choices of these manifolds are exponentially close at $\Delta^{i n}$ and even more so at $\Delta^{\text {out }}$ due to the exponential contraction during the passage.

2.2. Blow-up. In this section we define and describe the blow-up transformation. The basic observation is that the fold point $(0,0,0)$ is a more degenerate equilibrium point of system (2.5) than the other points of the critical manifold $S$. The 
linearization of system (2.5) at the origin has a triple zero eigenvalue while the linearization at the other points of the critical manifold $S$ has a double zero eigenvalue and one negative (resp., positive) eigenvalue for $x<0$ (resp., $x>0$ ).

The important insight in [6] is that blow-up techniques are the right tool to analyze nilpotent equilibria like the fold point, viewed as a degenerate equilibrium of the extended system (2.4). The blow-up method is essentially a clever coordinate transformation by which the degenerate equilibrium is "blown-up" to a two-sphere. In certain directions transverse to the sphere and even on the sphere, one gains enough hyperbolicity to allow a complete analysis by standard techniques. The technique is a generalization of the well known blow-up methods for degenerate equilibria of planar vector fields [5]. In the simplest situations this corresponds to blowing-up the degenerate equilibrium to the circle $r=0$ by rewriting the vector field in polar coordinates $(r, \vartheta) \in \mathbb{R} \times S^{1}$. The analysis is often simplified substantially by using a quasi-homogeneous blow-up, i.e. by using different powers (weights) of $r$ for different variables in the defining transformation.

The blow-up transformation for system (2.5) is

$$
x=\bar{r} \bar{x}, \quad y=\bar{r}^{2} \bar{y}, \quad \varepsilon=\bar{r}^{3} \bar{\varepsilon}
$$

with weights 1,2 , and 3 . We define $B=S^{2} \times[0, \rho]$, where the constant $\rho>0$ is related to $\varepsilon_{0}$ by $\varepsilon_{0}=\rho^{3}$. We consider the blow-up transformation as a mapping

$$
\Phi: B \rightarrow \mathbb{R}^{3}
$$

with $(\bar{x}, \bar{y}, \bar{\varepsilon}) \in S^{2}$. We choose $\rho>0$ sufficiently small such that system (2.4) is described by the canonical form (2.5) in the region $\Phi(B)$. We will be interested only in nonnegative values of $\bar{\varepsilon}$ and $\bar{r}$, but everything that follows makes sense for negative values as well, i.e., there are no technical problems at $\partial B$.

Let $X$ denote the vector field corresponding to (2.5). Since $X$ vanishes at the point $(0,0,0)$, there exists a vector field $\bar{X}$ on $B$ such that $\Phi_{*} \bar{X}=X$, where $\Phi_{*}$ is induced by $\Phi$. It remains to study the vector field $\bar{X}$ on the manifold $B$. Note that this suffices, since $\Phi(B)$ is a full neighborhood of the origin. In principle one could use spherical coordinates on $S^{2}$; however, this would lead to rather lengthy computations. It is natural and almost mandatory to use different charts for the manifold $B$ to simplify the analysis. One reason for this is that - as we will see later - the dynamics in the individual charts is very different.

We will now introduce the charts used later in this paper. Loosely speaking, we will define a chart $K_{2}$, which describes a neighborhood of the upper half-sphere defined by $\bar{\varepsilon}>0$, and charts $K_{1}$ and $K_{3}$ which describe neighborhoods of parts of the equator of $S^{2}$ which are needed in the analysis. In problems where a neighborhood of the whole equator needs to be analyzed, two further charts must be defined analogously. The subscripts in $K_{1}, K_{2}$, and $K_{3}$ denote the order in which the charts are used later.

The charts $K_{1}, K_{2}$, and $K_{3}$ are obtained by setting $\bar{y}=1, \bar{\varepsilon}=1$, and $\bar{x}=1$, respectively, in the blow-up transformation (2.6). The blow-up transformation in the charts $K_{i}, i=1,2,3$ is given by

$$
\begin{aligned}
& x=r_{1} x_{1}, \quad y=r_{1}^{2}, \quad \varepsilon=r_{1}^{3} \varepsilon_{1}, \\
& x=r_{2} x_{2}, \quad y=r_{2}^{2} y_{2}, \quad \varepsilon=r_{2}^{3},
\end{aligned}
$$




$$
x=r_{3}, \quad y=r_{3}^{2} y_{3}, \quad \varepsilon=r_{3}^{3} \varepsilon_{3}
$$

with coordinates $\left(x_{1}, r_{1}, \varepsilon_{1}\right) \in \mathbb{R}^{3},\left(x_{2}, y_{2}, r_{2}\right) \in \mathbb{R}^{3}$, and $\left(r_{3}, y_{3}, \varepsilon_{3}\right) \in \mathbb{R}^{3}$. The point $(0,0,0)$ is blown-up to the plane $r_{i}=0, i=1,2,3$. In our analysis we will need to change coordinates between these charts on their overlap domains. A simple computation gives the following lemma.

Lemma 2.2. Let $\kappa_{12}$ denote the change of coordinates from $K_{1}$ to $K_{2}$. Then $\kappa_{12}$ is given by

$$
x_{2}=x_{1} \varepsilon_{1}^{-1 / 3}, \quad y_{2}=\varepsilon_{1}^{-2 / 3}, \quad r_{2}=r_{1} \varepsilon_{1}^{1 / 3} \quad \text { for } \varepsilon_{1}>0,
$$

and $\kappa_{12}^{-1}$ is given by

$$
x_{1}=x_{2} y_{2}^{-1 / 2}, \quad r_{1}=r_{2} y_{2}^{1 / 2}, \quad \varepsilon_{1}=y_{2}^{-3 / 2} \quad \text { for } y_{2}>0 .
$$

Let $\kappa_{23}$ denote the change of coordinates from $K_{2}$ to $K_{3}$. Then $\kappa_{23}$ is given by

$$
r_{3}=r_{2} x_{2}, \quad y_{3}=y_{2} x_{2}^{-2}, \quad \varepsilon_{3}=x_{2}^{-3} \quad \text { for } x_{2}>0,
$$

and $\kappa_{23}^{-1}$ is given by

$$
x_{2}=\varepsilon_{3}^{-1 / 3}, \quad y_{2}=y_{3} \varepsilon_{3}^{-2 / 3}, \quad r_{2}=r_{3} \varepsilon_{3}^{1 / 3} \text { for } \varepsilon_{3}>0 .
$$

The above constructions make perfect sense if restricted to $B$. We introduce the following notation: $\bar{P}$ denotes an object in the blow-up which corresponds to an object $P$ in the original problem. If $\bar{P}$ is described in one of the charts, then $P_{i}$ denotes the object in chart $K_{i}, i=1,2,3$. This notation is used only when necessary, mostly to denote various invariant manifolds.

Remark 2.2. In the work of Dumortier and Roussarie the chart $K_{2}$ corresponding to a directional blow-up in the direction of $\varepsilon$ is called family rescaling, and charts used near the equator are called phase directional rescaling.

2.3. Blow-up of (1.2) with $\varepsilon=\mathbf{0}$. It is instructive to recall how the usual blow-up method applies to the layer problem, i.e., system (2.5) with $\varepsilon=0$. Setting $\bar{\varepsilon}=0$ in (2.6) defines a (planar, polar) blow-up of the degenerate equilibrium at the origin. To see this, note that $B \cap\{\bar{\varepsilon}=0\}=S^{1} \times[0, \rho]$, where $S^{1}=\left\{(\bar{x}, \bar{y}, 0) \in S^{2}\right\}$. Due to the equation $\varepsilon^{\prime}=0$, the set $S^{1} \times[0, \rho]$ is invariant for $\bar{X}$, which, restricted to $S^{1} \times[0, \rho]$, is the blow-up of (1.2) with $\varepsilon=0$.

Let $\bar{X}_{0}=\left.\bar{X}\right|_{S^{1} \times[0, \rho]}$. Figure 2.2 shows the phase portrait of $\bar{X}_{0}$. We briefly describe this phase portrait, referring the reader to the sections on charts $K_{1}$ and $K_{3}$ for technical details. On the invariant circle $S^{1}$, there are four equilibria: $p_{a}, p_{r}$, $q_{\text {in }}, q_{\text {out }}$. These equilibria are hyperbolic for the flow on $S^{1}$, the points $p_{a}$ and $q_{\text {out }}$ are attracting, and $p_{r}$ and $q_{i n}$ are repelling. The points $p_{a}$ and $p_{r}$ are end points of the blown-up critical manifolds $\bar{S}_{a}$ and $\bar{S}_{r}$, which are lines of equilibria for $\bar{X}_{0}$. Hence the radial direction is nonhyperbolic at $p_{a}$ and $p_{r}$. The points $q_{i n}$ and $q_{\text {out }}$ are the intersection points of $S^{1}$ with the blow-up of the critical fiber. These points are hyperbolic in the radial direction.

2.4. Dynamics in chart $\boldsymbol{K}_{\mathbf{2}}$. The dynamics of the blown-up vector field $\bar{X}$ in a neighborhood of the upper half-sphere is studied in chart $K_{2}$. The transformation (2.9) is just a rescaling of $(x, y)$, since $r_{2}=\varepsilon^{1 / 3}$. By inserting (2.9) into system (2.5) we obtain the vector field $\bar{X}$ in chart $K_{2}$. Since $r_{2}^{\prime}=0$, this blown-up system is still a 


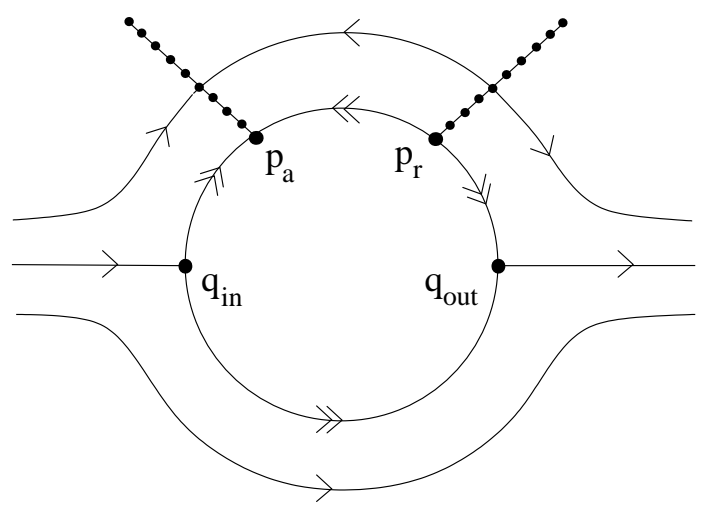

FIG. 2.2. Phase portrait of the blown-up vector field for $\bar{\varepsilon}=0$.

family of planar vector fields with parameter $r_{2}$. We now desingularize the equations by rescaling time $t_{2}:=r_{2} t$, so that the factor $r_{2}$ disappears. This desingularization is necessary to obtain a nontrivial flow on the blown-up locus $r_{2}=0$. We obtain

$$
\begin{aligned}
& x_{2}^{\prime}=x_{2}^{2}-y_{2}+O\left(r_{2}\right), \\
& y_{2}^{\prime}=-1+O\left(r_{2}\right), \\
& r_{2}^{\prime}=0,
\end{aligned}
$$

where' denotes differentiation with respect to $t_{2}$.

Remark 2.3. The rescaled form (2.15) of the original problem plays a crucial role in all approaches to the fold point by means of asymptotic expansions; e.g. [12], [18], and [20]. In these investigations solutions of (2.15) are used as inner solutions connecting (matching) solutions obtained as perturbations of the reduced problem to solutions obtained as solutions of the layer problem.

We first consider the case $r_{2}=0$, which gives

$$
\begin{aligned}
& x_{2}^{\prime}=x_{2}^{2}-y_{2}, \\
& y_{2}^{\prime}=-1 .
\end{aligned}
$$

This is a Riccati equation whose solutions can be expressed in terms of special functions. The relevant results can be found in [18, pp. 68-72]. Here we restate the results needed in our analysis. For the sake of readability we omit the subscript 2 of the variables.

Proposition 2.3 (see [18]). The Riccati equation (2.16) has the following properties:

1. Every orbit has a horizontal asymptote $y=y_{r}$, where $y_{r}$ depends on the orbit such that $x \rightarrow \infty$ as $y$ approaches $y_{r}$ from above.

2. There exists a unique orbit $\gamma_{2}$ which can be parametrized as $(x, s(x)), x \in \mathbb{R}$ and is asymptotic to the left branch of the parabola $x^{2}-y=0$ for $x \rightarrow-\infty$. The orbit $\gamma_{2}$ has a horizontal asymptote $y=-\Omega_{0}<0$ such that $x \rightarrow \infty$ as $y$ approaches $-\Omega_{0}$ from above.

3. The function $s(x)$ has the asymptotic expansions

$$
s(x)=x^{2}+\frac{1}{2 x}+O\left(\frac{1}{x^{4}}\right), \quad x \rightarrow-\infty,
$$




$$
s(x)=-\Omega_{0}+\frac{1}{x}+O\left(\frac{1}{x^{3}}\right), \quad x \rightarrow \infty .
$$

4. All orbits to the right of $\gamma_{2}$ are backward asymptotic to the right branch of the parabola $x^{2}-y=0$.

5. All orbits to the left of $\gamma_{2}$ have a horizontal asymptote $y=y_{l}>y_{r}$, where $y_{l}$ depends on the orbit, such that $x \rightarrow-\infty$ as $y$ approaches $y_{l}$ from below.

Remark 2.4. The constant $\Omega_{0}$ is the smallest positive zero of

$$
J_{-1 / 3}\left(2 z^{3 / 2} / 3\right)+J_{1 / 3}\left(2 z^{3 / 2} / 3\right),
$$

where $J_{-1 / 3}$ (resp., $J_{1 / 3}$ ) are Bessel functions of the first kind [18].

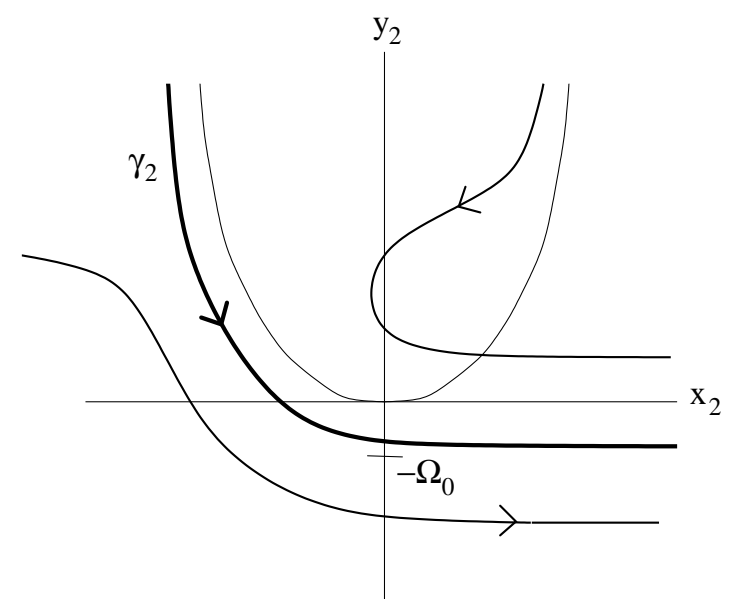

FIG. 2.3. Solutions of the Riccati equation (2.16).

The assertions of Proposition 2.3 are illustrated in Figure 2.3. We will see that the orbit $\bar{\gamma}$ corresponding to the special solution $\gamma_{2}$ is backward asymptotic to the equilibrium $p_{a}$ on the equator of $S^{2}$. The importance of the orbit $\bar{\gamma}$ is that it "leads" the incoming attracting slow manifold across the upper half of the sphere $S^{2}$ to the point $q_{\text {out }}$ from where take-off in the direction of the fast flow occurs.

We need to describe the transition map for (2.15) within a bounded domain $D_{2}$. Within such a domain we can deduce properties of the flow of (2.15) from Proposition 2.3 by using regular perturbation theory. A detailed study of the effect of the $O\left(r_{2}\right)$ perturbations outside $D_{2}$, i.e., close to infinity, will be carried out in the charts $K_{1}$ and $K_{3}$. For $\delta>0$ we define the following sections:

$$
\Sigma_{2}^{i n}=\left\{\left(x_{2}, y_{2}, r_{2}\right): y_{2}=\delta^{-2 / 3}\right\}, \quad \Sigma_{2}^{o u t}=\left\{\left(x_{2}, y_{2}, r_{2}\right): x_{2}=\delta^{-1 / 3}\right\} .
$$

Let $\Pi_{2}$ be the transition map of the flow (2.15) from $\Sigma_{2}^{i n}$ to $\Sigma_{2}^{o u t}$. Let $q_{0}=\gamma_{2} \cap \Sigma_{2}^{i n}$. Proposition 2.4. The transition map $\Pi_{2}$ has the following properties:

1.

$$
\Pi_{2}\left(q_{0}\right)=\left(\delta^{-1 / 3},-\Omega_{0}+\delta^{1 / 3}+O(\delta), 0\right) .
$$

2. A neighborhood of $q_{0}$ is mapped diffeomorphically onto a neighborhood of $\Pi_{2}\left(q_{0}\right)$.

Proof. The proof follows directly from Proposition 2.3 and regular perturbation theory. 


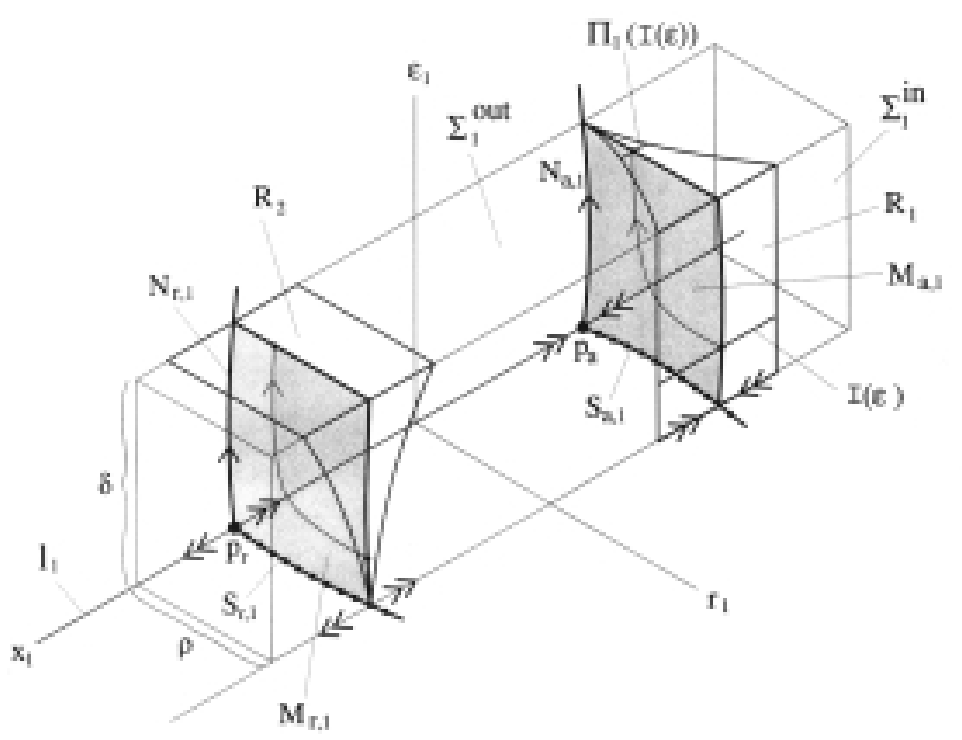

FIG. 2.4. Geometry and dynamics in chart $K_{1}$.

2.5. Dynamics in chart $\boldsymbol{K}_{\mathbf{1}}$. Chart $K_{1}$ is used to analyze the dynamics of the blown-up vector field $\bar{X}$ in a neighborhood of the equator containing the equilibria $p_{a}$ and $p_{r}$. By inserting (2.8) into system (2.5), we obtain the vector field $\bar{X}$ in chart $K_{1}$. We desingularize the blown-up vector field $\bar{X}$ by dividing by $r_{1}$. This gives

$$
\begin{aligned}
x_{1}^{\prime} & =-1+x_{1}^{2}+\frac{1}{2} \varepsilon_{1} x_{1}+O\left(r_{1}\right), \\
r_{1}^{\prime} & =\frac{1}{2} r_{1} \varepsilon_{1}\left(-1+O\left(r_{1}\right)\right), \\
\varepsilon_{1}^{\prime} & =\frac{3}{2} \varepsilon_{1}^{2}\left(1+O\left(r_{1}\right)\right),
\end{aligned}
$$

where ${ }^{\prime}$ denotes differentiation with respect to a rescaled time variable $t_{1}$.

Remark 2.5. The equation for $\varepsilon_{1}^{\prime}$ is obtained from the equation $\varepsilon^{\prime}=0$, which implies the relation $3 r_{1}^{2} r_{1}^{\prime} \varepsilon_{1}+r_{1}^{3} \varepsilon_{1}^{\prime}=0$. Hence $\varepsilon=r_{1}^{3} \varepsilon_{1}$ is a constant of motion in chart $K_{1}$. Nevertheless, we will see that it is useful to treat the blown-up system as a three-dimensional problem. This seemingly artificial construction is actually crucial for the whole approach.

Remark 2.6. For $r_{1}>0$, system (2.17) has the same orbits as the blown-up vector field $\bar{X}$ with the corresponding solutions having a different time parametrization. Since we deal only with transition maps between sections, time parametrization of solutions has no significance to our analysis.

System (2.17) has two invariant subspaces, namely, the plane $r_{1}=0$ and the plane $\varepsilon_{1}=0$. Their intersection is the invariant line $l_{1}:=\left\{\left(x_{1}, 0,0\right): x_{1} \in \mathbb{R}\right\}$; see Figure 2.4. The dynamics on $l_{1}$ is governed by $x_{1}^{\prime}=-1+x_{1}^{2}$. There are two 
equilibria $p_{a}=(-1,0,0)$ and $p_{r}=(1,0,0)$. For the flow on the line $l_{1}$ both points are hyperbolic, the relevant eigenvalue is -2 for $p_{a}$ and 2 for $p_{r}$, i.e., $p_{a}$ is attracting and $p_{r}$ is repelling. The dynamics in the invariant plane $\varepsilon_{1}=0$ is governed by

$$
\begin{aligned}
& x_{1}^{\prime}=-1+x_{1}^{2}+O\left(r_{1}\right), \\
& r_{1}^{\prime}=0 .
\end{aligned}
$$

This system has a normally hyperbolic curve $S_{a, 1}$ of equilibria emanating from $p_{a}$ and a curve $S_{r, 1}$ of equilibria emanating from $p_{r}$; see Figure 2.4. For $r_{1}$ small, this follows from the implicit function theorem. Actually, $S_{a, 1}$ and $S_{r, 1}$ are precisely the branches of the critical manifold $S$ described in section 2.1; this also explains the notation. Along the curve $S_{a, 1}$ the linearization of (2.18) has one zero eigenvalue, the other eigenvalue is negative and close to -2 for $r_{1}$ small. Along $S_{r, 1}$ the situation is similar; however, the nonzero eigenvalue is positive and close to 2 for $r_{1}$ small.

Remark 2.7. Equation (2.18) is the directional (in the positive $y$-direction) blowup of the fold point $(0,0)$ of the layer problem, i.e., system (1.2) with $\varepsilon=0$. The line $l_{1}=0$ corresponds to the fold point. We have gained normal hyperbolicity of the lines of equilibria $S_{a, 1}$ (resp., $S_{r, 1}$ ) at the points $p_{a}$ (resp., $p_{r}$ ) due to the blow-up (compare Figure 2.2).

The dynamics in the invariant plane $r_{1}=0$ is governed by

$$
\begin{aligned}
x_{1}^{\prime} & =-1+x_{1}^{2}+\frac{1}{2} \varepsilon_{1} x_{1}, \\
\varepsilon_{1}^{\prime} & =\frac{3}{2} \varepsilon_{1}^{2} .
\end{aligned}
$$

We recover the equilibria points $p_{a}$ and $p_{r}$; however, there exists an additional zero eigenvalue due to the second equation. The corresponding eigenvector is $(-1,4)$ at both equilibria. Hence, there exist one-dimensional center manifolds $N_{a, 1}$ at $p_{a}$ and $N_{r, 1}$ at $p_{r}$ along which $\varepsilon_{1}$ increases for $\varepsilon_{1}>0$. Note that the branch of the attracting center manifold $N_{a, 1}$ at $p_{a}$ in the half space $\varepsilon_{1}>0$ is unique, while the repelling center manifold $N_{r, 1}$ at $p_{r}$ in the half space $\varepsilon_{1}>0$ is not unique; see Figure 2.4. We collect the information we have obtained so far in the following lemma.

Lemma 2.5. The linearization of system (2.17) at $p_{j}, j=a, r$ has the following real eigenvalues: $\lambda_{1}=-2$ at $p_{a}$ and $\lambda_{1}=2$ at $p_{r}$ with eigenvector $(1,0,0)$ corresponding to the flow on $l_{1}, \lambda_{2}=0$ with an eigenvector tangent to $S_{j, 1}$, and $\lambda_{3}=0$ with an eigenvector $(-1,0,4)$ corresponding to the center direction in the invariant plane $r_{1}=0$.

We restrict our attention to the set

$$
D_{1}:=\left\{\left(x_{1}, r_{1}, \varepsilon_{1}\right): x_{1} \in \mathbb{R}, 0 \leq r_{1} \leq \rho, 0 \leq \varepsilon_{1} \leq \delta\right\}
$$

where $\rho>0$ is the constant defining the sections $\Delta^{i n}$ and $\Delta^{\text {out }}$ in section 2.1 and $\delta>0$ is the constant defining the sections $\Sigma_{2}^{\text {in }}$ and $\Sigma_{2}^{\text {out }}$ in section 2.4. Note that all objects defined later extend smoothly to negative values of $r_{1}$ and $\varepsilon_{1}$; i.e., there are no problems due to the boundaries $r_{1}=0$ and $\varepsilon_{1}=0$. We have the following result.

Proposition 2.6. For $\rho, \delta$ sufficiently small the following assertions hold for system (2.17):

1. There exists an attracting two-dimensional $C^{k}$-center manifold $M_{a, 1}$ at $p_{a}$ which contains the line of equilibria $S_{a, 1}$ and the center manifold $N_{a, 1}$. In $D_{1}$ the manifold $M_{a, 1}$ is given as a graph $x_{1}=h_{a}\left(r_{1}, \varepsilon_{1}\right)$. The branch of $N_{a, 1}$ in $r_{1}=0, \varepsilon_{1}>0$ is unique. 
2. There exists a repelling two-dimensional $C^{k}$-center manifold $M_{r, 1}$ at $p_{r}$ which contains the line of equilibria $S_{r, 1}$ and the center manifold $N_{r, 1}$. In $D_{1}$ the manifold $M_{r, 1}$ is given as a graph $x_{1}=h_{r}\left(r_{1}, \varepsilon_{1}\right)$. The branch of $N_{r, 1}$ in $r_{1}=0, \varepsilon_{1}>0$ is not unique.

3. There exists a stable invariant foliation $\mathcal{F}^{s}$ with base $M_{a, 1}$ and one-dimensional fibers. For any $c>-2$ there exists a choice of positive $\rho$ and $\delta$ such that the contraction along $\mathcal{F}^{s}$ during a time interval $[0, T]$ is stronger than $e^{c T}$.

4. There exists an unstable invariant foliation $\mathcal{F}^{u}$ with base $M_{r, 1}$ and onedimensional fibers. For any $c<2$ there exists a choice of positive $\rho$ and $\delta$ such that the expansion along $\mathcal{F}^{u}$ during a time interval $[0, T]$ is stronger than $e^{c T}$.

5. The unique branch of $N_{a, 1}$ in $r_{1}=0, \varepsilon_{1}>0$ is equal to $\gamma_{1}:=\kappa_{12}^{-1}\left(\gamma_{2}\right)$, wherever $\kappa_{12}^{-1}$ is defined, i.e., along the part of $\gamma_{2}$ corresponding to $y_{2}>0$.

Proof. Assertions (1)-(4) follow from Lemma 2.5 and center manifold theory; see, e.g., [4], [10]. Proposition 2.3 and the coordinate transformation (2.12) imply that $\kappa_{12}^{-1}\left(\gamma_{2}\right)$ has the expansion

$$
\left(x_{2}\left(x_{2}^{2}+\frac{1}{2 x_{2}}+O\left(\frac{1}{x_{2}^{4}}\right)\right)^{-1 / 2}, 0,\left(x_{2}^{2}+\frac{1}{2 x_{2}}+O\left(\frac{1}{x_{2}^{4}}\right)\right)^{-3 / 2}\right)
$$

as $x_{2} \rightarrow-\infty$. Expanding these terms in powers of $x_{2}$ shows that $\kappa_{12}^{-1}\left(\gamma_{2}\right)$ converges to $p_{a}$ tangent to the center-direction $(-1,0,4)$ as $x_{2} \rightarrow-\infty$. This and the uniqueness of the branch of $N_{a, 1}$ in $r_{1}=0, \varepsilon_{1}>0$ imply assertion (5).

Remark 2.8. Clearly, the center manifold $M_{a, 1}$ in chart $K_{1}$ corresponds to a locally invariant manifold $\bar{M}_{a}$ of the blown-up vector field $\bar{X}$. The importance of assertion (5) in the above proposition is that it allows us to track the manifold $\bar{M}_{a}$ as it moves across the sphere $S^{2}$ guided by the special orbit $\bar{\gamma}$ corresponding to the solution $\gamma_{2}$ of the Riccati equation.

We now define the following sections:

$$
\Sigma_{1}^{\text {in }}:=\left\{\left(x_{1}, r_{1}, \varepsilon_{1}\right) \in D_{1}: r_{1}=\rho\right\}, \quad \Sigma_{1}^{o u t}:=\left\{\left(x_{1}, r_{1}, \varepsilon_{1}\right) \in D_{1}: \varepsilon_{1}=\delta\right\} .
$$

Remark 2.9. Note that $\Sigma_{1}^{i n}$ maps under the blow-up transformation (2.8) to $\Delta^{i n}$ and $\Sigma_{1}^{\text {out }}$ maps under the coordinate transformation (2.11) to $\Sigma_{2}^{i n}$. An important part of our description of the flow near the fold is the description of the transition map from $\Sigma_{1}^{i n}$ to $\Sigma_{1}^{o u t}$ near the center manifolds $M_{a, 1}$ and $M_{r, 1}$. Since the neighborhood of $M_{a, 1}$ corresponds to the neighborhood of the attracting branch of the slow manifold of (1.2), we are more interested in understanding the dynamics near $M_{a, 1}$. Yet the analysis of the two cases is very similar, so we handle them simultaneously.

Let $R_{1}$ be the rectangle in $\Sigma_{1}^{i n}$ defined by $\left|1+x_{1}\right| \leq \beta_{1}$, and let $R_{2}$ be the rectangle in $\Sigma_{1}^{\text {out }}$ defined by $\left|1-x_{1}\right| \leq \beta_{1}$ for sufficiently small $\beta_{1}>0$. The constants $\rho, \delta$, and $\beta_{1}$ can be chosen such that $M_{a, 1} \cap \Sigma_{1}^{\text {in }} \subset R_{1}$ and $M_{r, 1} \cap \Sigma_{1}^{\text {out }} \subset R_{2}$. For $0 \leq \tilde{\varepsilon} \leq \delta$ and $0 \leq \tilde{r} \leq \rho$, let $I_{a}(\tilde{\varepsilon})$ be the line $R_{1} \cap\left\{\varepsilon_{1}=\tilde{\varepsilon}\right\}$ and $I_{r}(\tilde{r})$ be the line $R_{2} \cap\left\{r_{1}=\tilde{r}\right\}$.

In the neighborhood of $p_{j}, j=a, r$, the flow of (2.17) carries $\Sigma_{1}^{i n}$ to $\Sigma_{1}^{\text {out }}$. Let $\Pi_{1}: \Sigma_{1}^{\text {in }} \rightarrow \Sigma_{1}^{\text {out }}$ be the transition map defined by the flow of (2.17). The map $\Pi_{1}$ is well defined on $R_{1}$, at least for small enough values of $\rho, \delta$, and $\beta_{1}$. The map $\Pi_{1}$ is defined in a wedge-shaped set in $\Sigma_{1}^{i n}$ around $M_{r, 1}$ that shrinks to $S_{r, 1}$ for $\varepsilon_{1} \rightarrow 0$. The reason for this difference is that $M_{a, 1}$ is attracting and $M_{r, 1}$ is repelling. We have the following estimate of transition times. 
Lemma 2.7. The transition time $T$ of a solution of system (2.17) from a point $p=\left(x_{1}, \rho, \varepsilon_{1}\right) \in \Sigma_{1}^{\text {in }}$ to the point $\Pi_{1}(p) \in \Sigma_{1}^{\text {out }}$ satisfies

$$
T=\frac{2}{3}\left(\frac{1}{\varepsilon_{1}}-\frac{1}{\delta}\right)(1+O(\rho))
$$

Proof. The evolution of $\varepsilon_{1}$ determines the transition time of solutions from $\Sigma_{1}^{i n}$ to $\Sigma_{1}^{\text {out }}$. The relevant equation is

$$
\varepsilon_{1}^{\prime}=\frac{3}{2} \varepsilon_{1}^{2}\left(1-O\left(r_{1}\right)\right) .
$$

The result follows immediately by integrating (2.21).

Proposition 2.8. For $\rho, \delta$, and $\beta_{1}$ sufficiently small the transition map $\Pi_{1}$ : $\Sigma_{1}^{i n} \rightarrow \Sigma_{1}^{o u t}$ defined by the flow of system (2.17) has the following properties:

1. $\Pi_{1}\left(R_{1}\right)$ is a wedge-like region in $\Sigma_{1}^{\text {out }} . \Pi_{1}^{-1}\left(R_{2}\right)$ is a wedge-like region in $\Sigma_{1}^{\text {in }}$.

2. More precisely, for fixed $c<2$ there exists a constant $K$ depending on the constants $c, \rho, \delta$, and $\beta_{1}$ such that

(i) for $\varepsilon_{1} \in(0, \delta]$ the map $\Pi_{1} \mid I_{a}\left(\varepsilon_{1}\right)$ is a contraction with contraction rate bounded by $K e^{-\frac{2 c}{3}\left(\frac{1}{\varepsilon_{1}}-\frac{1}{\delta}\right)}$.

(ii) for $r_{1} \in(0, \rho]$ the map $\Pi_{1}^{-1} \mid I_{r}\left(r_{1}\right)$ is a contraction with contraction rate bounded by $K e^{-\frac{2 c}{3}\left(\frac{\rho^{3}}{r_{1}^{3} \delta}-\frac{1}{\delta}\right)}$.

Proof. The assertions follow from Proposition 2.6 and Lemma 2.7. The estimate for the contraction rate of $\Pi_{1}^{-1}$ in the second assertion uses the identity $\varepsilon_{1} \rho^{3}=\delta r_{1}^{3}$ for $p=\left(x_{1, \text { in }}, \rho, \varepsilon_{1}\right)$ and $\Pi_{1}(p)=\left(x_{1, \text { out }}, r_{1}, \delta\right)$ to express the transition time in terms of $r_{1}$.

All our results concerning the dynamics in chart $K_{1}$ are illustrated in Figure 2.4.

2.6. Dynamics in chart $\boldsymbol{K}_{\mathbf{3}}$. We use chart $K_{3}$ to analyze the dynamics of the blown-up vector field $\bar{X}$ in a neighborhood of the equator containing the point $q_{\text {out }}$. Applying transformation (2.10) to system (2.5) and desingularizing by dividing out the factor $r_{3}$, we obtain

$$
\begin{aligned}
& r_{3}^{\prime}=r_{3} F\left(r_{3}, y_{3}, \varepsilon_{3}\right), \\
& y_{3}^{\prime}=\varepsilon_{3}\left(-1+O\left(r_{3}\right)\right)-2 y_{3} F\left(r_{3}, y_{3}, \varepsilon_{3}\right), \\
& \varepsilon_{3}^{\prime}=-3 \varepsilon_{3} F\left(r_{3}, y_{3}, \varepsilon_{3}\right),
\end{aligned}
$$

where $F\left(r_{3}, y_{3}, \varepsilon_{3}\right):=1-y_{3}+O\left(r_{3}\right)$. The planes $\varepsilon_{3}=0$ and $r_{3}=0$ and the $y_{3}$-axis are invariant under the flow of (2.22).

Lemma 2.9. The point $q_{\text {out }}=(0,0,0)$ is a hyperbolic equilibrium of system (2.22) with eigenvalues: $\lambda_{1}=1$ with eigenvector $(1,0,0)$ corresponding to the flow in $\varepsilon_{3}=0, \lambda_{2}=-2$ with eigenvector $(0,1,0)$ corresponding to the flow on the $y_{3}$-axis, and $\lambda_{3}=-3$ with eigenvector $(0,1,1)$ corresponding to the flow in $r_{3}=0$.

Proof. Computation.

Now we transform the part of the special orbit $\gamma_{2}$ (introduced in Proposition 2.3) corresponding to $x_{2}>0$ to chart $K_{3}$; i.e., we define $\gamma_{3}:=\kappa_{23}\left(\gamma_{2}\right)$.

Lemma 2.10. The orbit $\gamma_{3}$ lies in the plane $r_{3}=0$, converges to qout as $\varepsilon_{3} \rightarrow 0$, and is tangent at $q_{\text {out }}$ to the vector $(0,1,0)$.

Proof. The coordinate transformation (2.13) and assertion (3) from Proposition 2.3 imply that the orbit $\gamma_{3}$ has the expansion $\left(0,-\Omega_{0} \varepsilon_{3}^{2 / 3}+\varepsilon_{3}+O\left(\varepsilon_{3}^{5 / 3}\right), \varepsilon_{3}\right)$ as $\varepsilon_{3} \rightarrow 0$. The lemma follows. 
Lemma 2.10 implies that parts of the manifold $\bar{M}_{a}$ corresponding to $\bar{r}>0$ come close to the equilibrium $q_{\text {out }}$. Hence, we need a precise description of the dynamics of system (2.22) close to $q_{\text {out }}$. This is a somewhat delicate problem because of the resonance $\lambda_{2}=\lambda_{1}+\lambda_{3}$, which implies that there exists no smooth transformation of the nonlinear flow to the flow of the corresponding linearization. It turns out that, due to the simple form of the equations, it is quite easy to work out the lowest order approximation of the flow.

For the description of the flow in a neighborhood of $q_{\text {out }}$ we define sections $\Sigma_{3}^{i n}$ and $\Sigma_{3}^{\text {out }}$ as follows:

$$
\begin{aligned}
& \Sigma_{3}^{\text {in }}=\left\{\left(r_{3}, y_{3}, \varepsilon_{3}\right): r_{3} \in[0, \rho], y_{3} \in\left[-\beta_{3}, \beta_{3}\right], \varepsilon_{3}=\delta\right\} \\
& \Sigma_{3}^{\text {out }}=\left\{\left(r_{3}, y_{3}, \varepsilon_{3}\right): r_{3}=\rho, y_{3} \in\left[-\beta_{3}, \beta_{3}\right], \varepsilon_{3} \in[0, \delta]\right\}
\end{aligned}
$$

where $\rho$ and $\delta$ are the same constants as before, and $\beta_{3}>0$ is sufficiently small; see Figure 2.5.

Let $\Pi_{3}$ be the transition map from $\Sigma_{3}^{i n}$ to $\Sigma_{3}^{\text {out }}$. Our goal is to obtain a formula for the map $\Pi_{3}$. Before stating the relevant result we need to discuss the structure of (2.22) in more detail. We first divide $(2.22)$ by the factor $F\left(r_{3}, y_{3}, \varepsilon_{3}\right)$, which is close to one near $q_{\text {out }}$, and obtain

$$
\begin{aligned}
& r_{3}^{\prime}=r_{3}, \\
& y_{3}^{\prime}=-2 y_{3}-\frac{\varepsilon_{3}}{1-y_{3}}+r_{3} \varepsilon_{3} G\left(r_{3}, y_{3}, \varepsilon_{3}\right), \\
& \varepsilon_{3}^{\prime}=-3 \varepsilon_{3},
\end{aligned}
$$

where $G\left(r_{3}, y_{3}, \varepsilon_{3}\right)$ is a $C^{k}$-function. Consider (2.23) with $r_{3}=0$, namely,

$$
\begin{aligned}
& y_{3}^{\prime}=-2 y_{3}-\frac{\varepsilon_{3}}{1-y_{3}}, \\
& \varepsilon_{3}^{\prime}=-3 \varepsilon_{3} .
\end{aligned}
$$

By construction, system (2.24) is, up to rescaling of time, the Riccati equation (2.16) transformed to $K_{3}$. The corresponding linearization has eigenvalues $\lambda_{2}=-2$ and $\lambda_{3}=-3$; hence, (2.16) can be linearized by a near identity transformation of the form

$$
y_{3}=\psi\left(\tilde{y}_{3}, \varepsilon_{3}\right),
$$

where the function $\psi$ is $C^{k}$ smooth and $\psi\left(\tilde{y}_{3}, \varepsilon_{3}\right)=\tilde{y}_{3}+{ }_{\sim} O\left(\tilde{y}_{3} \varepsilon_{3}\right)$; see [22]. The corresponding inverse transformation is denoted by $\tilde{y}_{3}=\tilde{\psi}\left(y_{3}, \varepsilon_{3}\right)=y_{3}+O\left(y_{3} \varepsilon_{3}\right)$. Under the transformation (2.25) system (2.23) becomes

$$
\begin{aligned}
& r_{3}^{\prime}=r_{3}, \\
& \tilde{y}_{3}^{\prime}=-2 \tilde{y}_{3}-\varepsilon_{3}+r_{3} \varepsilon_{3} H\left(r_{3}, \tilde{y}_{3}, \varepsilon_{3}\right), \\
& \varepsilon_{3}^{\prime}=-3 \varepsilon_{3},
\end{aligned}
$$

with a $C^{k}$-function $H$. We have the following result.

Proposition 2.11. The transition map $\Pi_{3}$ for system (2.22) has the form

$$
\Pi_{3}\left(r_{3}, y_{3}, \delta\right)=\left(\begin{array}{c}
\rho \\
\Pi_{32}\left(r_{3}, y_{3}, \delta\right) \\
\left(\frac{r_{3}}{\rho}\right)^{3} \delta
\end{array}\right)
$$


with $\Pi_{32}\left(r_{3}, y_{3}, \delta\right)$ given by

$$
\Pi_{32}\left(r_{3}, y_{3}, \delta\right)=\left(\tilde{\psi}\left(y_{3}, \delta\right)-\delta\right)\left(\frac{r_{3}}{\rho}\right)^{2}+O\left(r_{3}^{3} \ln r_{3}\right) .
$$

Proof. In the following we suppress the subscript 3 in system (2.26). Fix $\left(r_{i}, \tilde{y}_{i}, \delta\right) \in$ $\Sigma^{\text {in }}$ and $\left(\rho, \tilde{y}_{o}, \varepsilon_{o}\right) \in \Sigma^{o u t}$. Consider a solution $(r, \tilde{y}, \varepsilon)(t)$ of $(2.26)$ and $T>0$ such that $r(0)=r_{i}, r(T)=\rho, \tilde{y}(0)=\tilde{y}_{i}, \tilde{y}(T)=\tilde{y}_{o}, \varepsilon(0)=\delta, \varepsilon(T)=\varepsilon_{o}$. We will now compute $\left(T, \tilde{y}_{o}, \varepsilon_{o}\right)$ as a function of $\left(r_{i}, \tilde{y}_{i}\right)$. Equations $(2.26 \mathrm{a})$ and $(2.26 \mathrm{c})$ have explicit solutions $r=e^{t} r_{i}, \varepsilon=\delta e^{-3 t}$. The requirement $r(T)=\rho$ produces an expression for $T$, namely,

$$
T=\ln \left(\frac{\rho}{r_{i}}\right) .
$$

Let $z$ be a new coordinate defined by $\tilde{y}=e^{-2 t}\left(\tilde{y}_{i}-\delta+z\right)+\delta e^{-3 t}$. We get the following equation for $z$ :

$$
z^{\prime}=r_{i} H^{z}\left(z, r_{i}, \tilde{y}_{i}, t\right)
$$

where $H^{z}\left(z, r_{i}, \tilde{y}_{i}, t\right)=\delta H\left(e^{t} r_{i}, e^{-2 t}\left(\tilde{y}_{i}-\delta+z\right)+\delta e^{-3 t}, \delta e^{-3 t}\right)$. The transition time $T$ is still given by (2.27). Note that the function $H^{z}$ is uniformly bounded on the relevant domain. Using (2.28) we obtain $z(T)=r_{i} O(T)=O\left(r_{i} \ln \left(\frac{\rho}{r_{i}}\right)\right)$. It follows that

$$
\tilde{y}(T)=\left(\tilde{y}_{i}-\delta\right)\left(\frac{r_{i}}{\rho}\right)^{2}+O\left(\frac{r_{i}^{3}}{\rho^{2}} \ln \left(\frac{\rho}{r_{i}}\right)\right) .
$$

Hence

$$
\begin{aligned}
\Pi_{32}\left(r_{3}, y_{3}, \delta\right) & =\psi\left(\left(\tilde{\psi}\left(y_{3}, \delta\right)-\delta\right)\left(\frac{r_{3}}{\rho}\right)^{2}+O\left(r_{3}^{3} \ln r_{3}\right),\left(\frac{r_{3}}{\rho}\right)^{3} \delta\right) \\
& =\left(\tilde{\psi}\left(y_{3}, \delta\right)-\delta\right)\left(\frac{r_{3}}{\rho}\right)^{2}+O\left(r_{3}^{3} \ln r_{3}\right)
\end{aligned}
$$

and the result follows.

Remark 2.10. The following observation will be used later in this paper to obtain the leading order asymptotics of the extended slow manifold $S_{a, \varepsilon}$. The $y_{3}$ coordinate of the point where the special orbit $\gamma_{3}$ intersects the section $\Sigma_{3}^{i n}$ is $y_{3}^{*}=\delta^{2 / 3} s\left(\delta^{-1 / 3}\right)$ (see the proof of Lemma 2.10). By comparing the asymptotics of $\gamma_{3}$ and the exact solution of system (2.26) restricted to $r_{3}=0$, i.e., the Riccati equation written in the linearizing coordinates $\left(\tilde{y}_{3}, \varepsilon_{3}\right)$, it follows that $\tilde{\psi}\left(y_{3}^{*}, \delta\right)-\delta=-\Omega_{0} \delta^{2 / 3}$.

2.7. Phase portrait on the upper part of $S^{2}$. The sphere $S^{2}$ is invariant under the desingularization of the blown-up vector field $\bar{X}$. The equator $S^{1}$ is invariant. On $S^{1}$ there are four equilibria $p_{a}, p_{r}, q_{\text {in }}, q_{\text {out }}$. These equilibria are hyperbolic for the flow on $S^{1}$, the points $p_{a}$ and $q_{o u t}$ are attracting, and $p_{r}$ and $q_{i n}$ are repelling. All orbits in $S^{2,+}$ are forward asymptotic to $q_{\text {out }}$. The special orbit $\bar{\gamma}$ is backward asymptotic to $p_{a}$ and, as it arrives at $q_{o u t}$, it is tangent to $S^{1}$. Besides $\bar{\gamma}$ there exist two families of trajectories: backward asymptotic to $p_{r}$ or backward asymptotic to $q_{i n}$. The corresponding phase portrait of $S^{2}$ is shown in Figure 2.6. 


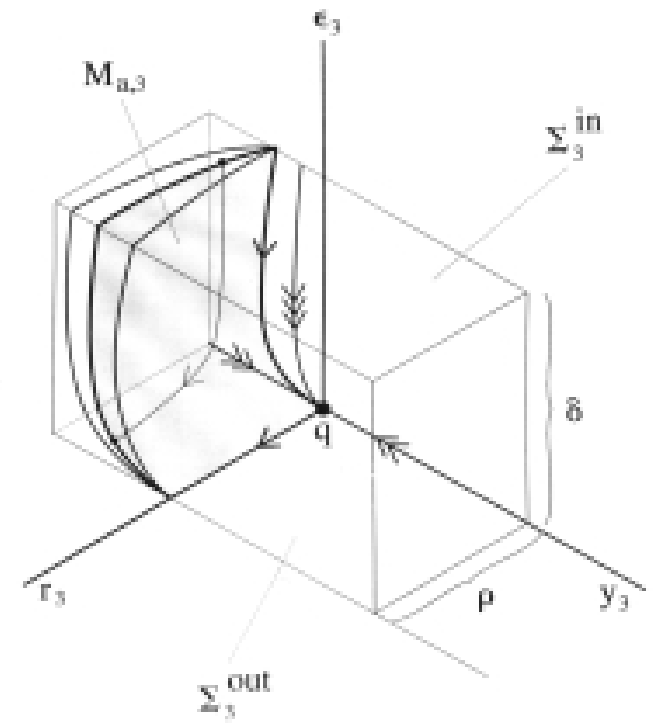

FIG. 2.5. Geometry and dynamics of system (2.22) near the equilibrium qout.

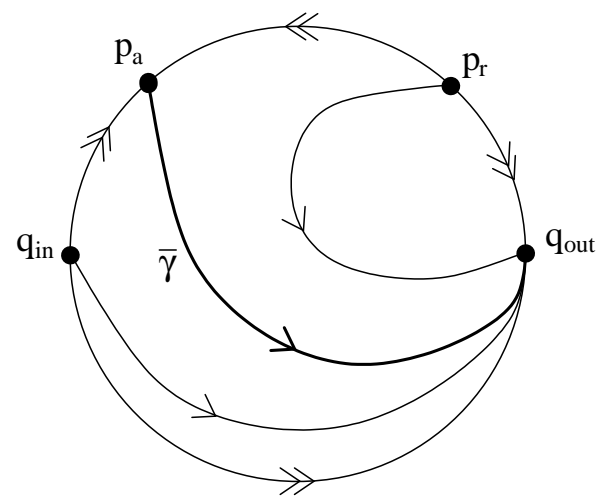

FIG. 2.6. Blow-up of a fold (jump) point restricted to $S^{2}$. 
2.8. Proof of the main result. In this section we prove Theorem 2.1 by combining the results obtained in the individual charts. The idea of the proof is to analyze the evolution of the center manifold $M_{a, 1}$ and the rectangle $R_{1}$ under the flow of the blown-up vector field $\bar{X}$. We denote the corresponding global invariant manifold by $\bar{M}_{a}$. The intersection of $\bar{M}_{a}$ with $S^{2++}$ is the special orbit $\bar{\gamma}$ connecting the equilibria $p_{a}$ and $q_{\text {out }}$. It follows that a trajectory starting in or nearby $\bar{M}_{a}$ will remain close to $\bar{\gamma}$ until it reaches the vicinity of $q_{\text {out }}$. There the trajectory follows the local dynamics near $q_{\text {out }}$ and is repelled in the unstable direction of $q_{\text {out }}$.

Proof of Theorem 2.1. We define the map $\Pi: \Sigma_{1}^{i n} \rightarrow \Sigma_{3}^{\text {out }}$ by

$$
\Pi:=\Pi_{3} \circ \kappa_{23} \circ \Pi_{2} \circ \kappa_{12} \circ \Pi_{1} .
$$

The map $\Pi$ is the transition map from $\Sigma_{1}^{i n}$ to $\Sigma_{3}^{o u t}$ for the flow induced by the blownup vector field $\bar{X}$ on $B$. We will analyze $\Pi\left(R_{1} \cap M_{a, 1}\right)$ and then use the fact that, by construction, the transition map $\pi$ is given by $\pi=\Phi \circ \Pi \circ \Phi^{-1}$ for $\varepsilon>0$.

It follows from Proposition 2.8 that $\Pi_{1}\left(R_{1} \cap M_{a, 1}\right) \subset \Sigma_{1}^{\text {out }}$ is a smooth curve transverse to the set $\left\{r_{1}=0\right\}$. It follows that $\kappa_{12}\left(\Pi_{1}\left(R_{1} \cap M_{a, 1}\right)\right)$ is a smooth curve transverse to the plane $\left\{r_{2}=0\right\}$. Proposition 2.4 implies that the image of this curve under $\Pi_{2}$ has the form $\left\{\left(\delta^{-1 / 3}, h_{2}^{\text {out }}\left(r_{2}\right), r_{2}\right): r_{2} \in\left[0, \rho \delta^{1 / 3}\right]\right\}$, where $h_{2}^{\text {out }}:\left[0, \rho \delta^{1 / 3}\right] \rightarrow \mathbb{R}$ is a smooth function. Under the transformation $\kappa_{23}$, this curve transforms to a smooth curve of the form $\left\{\left(r_{3}, h_{3}^{\text {in }}\left(r_{3}\right), \delta\right): r_{3} \in[0, \rho]\right\}$ with $\left(0, h_{3}^{\text {in }}(0), \delta\right)=\kappa_{23}\left(\gamma_{2} \cap \Sigma_{2}^{\text {out }}\right)$. Proposition 2.11 now implies that $\Pi\left(R_{1} \cap M_{a, 1}\right)$ has the form $\left\{\left(\rho, h_{3}^{\text {out }}\left(\varepsilon_{3}\right), \varepsilon_{3}\right): \varepsilon_{3} \in[0, \delta]\right\}$, where $h_{3}^{\text {out }}\left(\varepsilon_{3}\right)=O\left(\varepsilon_{3}^{2 / 3}\right)$. This proves assertion (1) of the theorem.

We now prove assertion (2). It follows from Proposition 2.8 that $\Pi_{1}\left(R_{1}\right)$ is a wedge-like region around $\Pi\left(R_{1} \cap M_{a, 1}\right)$ of width $O\left(e^{-c / \varepsilon_{1}}\right)$, where $c>0$ is some constant. Since $\kappa_{12}, \Pi_{2}$ and $\kappa_{23}$ are diffeomorphisms restricted to $\Sigma_{1}^{\text {out }}, \Sigma_{2}^{\text {in }}$, and $\Sigma_{2}^{\text {out }}$, respectively, it follows that $\kappa_{23} \circ \Pi_{2} \circ \kappa_{12} \circ \Pi_{1}\left(R_{1}\right)$ is also a wedge-like region of width $O\left(e^{-c / \varepsilon_{1}}\right)$ around $\kappa_{23} \circ \Pi_{2} \circ \kappa_{12} \circ \Pi_{1}\left(R_{1} \cap M_{a, 1}\right)$. Finally, we apply Proposition 2.11 to conclude that $\Pi\left(R_{1}\right)$ is a wedge-like region of width $O\left(e^{-c / \varepsilon_{1}}\right)$ around $\Pi\left(R_{1} \cap M_{a, 1}\right)$. The evolution of $R_{1}$ in the three charts is shown in Figure 2.7. Because $\varepsilon=\rho^{3} \varepsilon_{1}=$ $\rho^{3} \varepsilon_{3}$ is a constant of motion for the flow of $\bar{X}$, lines $\varepsilon_{1}=\varepsilon / \rho^{3}$ in $\Sigma_{1}^{i n}$ are mapped to lines $\varepsilon_{3}=\varepsilon / \rho^{3}$ in $\Sigma_{3}^{o u t}$. Restricted to such lines the map $\Pi$ is a contraction with contraction rate $O\left(e^{-c / \varepsilon_{1}}\right)$ for some $c>0$. Assertion (2) follows by applying the appropriate blow-down transformations.

The dynamics of the blown-up vector field $\bar{X}$, and in particular the center manifold $\bar{M}_{a}$, are shown in Figure 2.7.

Remark 2.11. Remark 2.10 implies that the function $h_{3}^{\text {out }}\left(\varepsilon_{3}\right)$ has the asymptotic expansion

$$
h_{3}^{\text {out }}\left(\varepsilon_{3}\right)=-\Omega_{0} \varepsilon_{3}^{2 / 3}+o\left(\varepsilon_{3}^{2 / 3}\right) .
$$

The corresponding expansion for the function $h(\varepsilon)$ in Theorem 2.1 is

$$
h(\varepsilon)=-\Omega_{0} \varepsilon^{2 / 3}+o\left(\varepsilon^{2 / 3}\right) .
$$

This result is well known; see, e.g., [18], where it is also shown that the next term in the expansion is $O(\varepsilon \ln \varepsilon)$. Our analysis, in particular the description of the map $\Pi_{3}$ in Proposition 2.11, shows that the occurrence of this term is due to the resonance $\lambda_{2}=\lambda_{1}+\lambda_{3}$ at the equilibrium $q_{\text {out }}$; see section 2.6. 


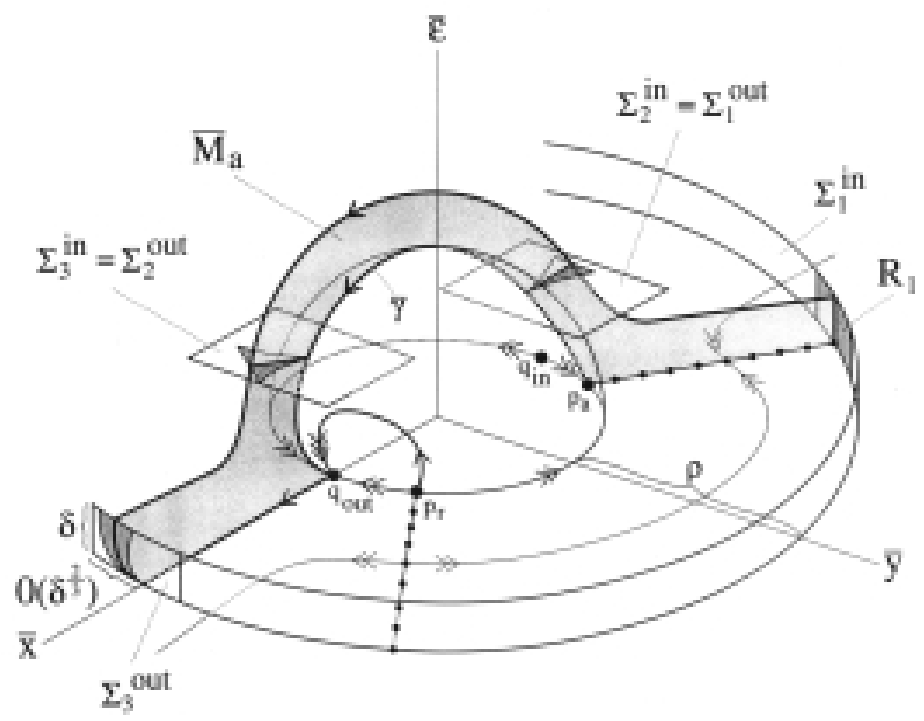

FIG. 2.7. Geometry and dynamics of the blown-up vector field $\bar{X}$.

\section{Canard point.}

3.1. Assumptions and results. In this section we consider a one parameter family of ODEs similar to (1.2), namely,

$$
\begin{aligned}
& x^{\prime}=f(x, y, \lambda, \varepsilon), \\
& y^{\prime}=\varepsilon g(x, y, \lambda, \varepsilon) .
\end{aligned}
$$

We assume that $\left(x_{0}, y_{0}\right)=(0,0)$ is a nondegenerate fold point of the critical manifold $f(x, y, \lambda, 0)=0$ for $\lambda_{0}=0$. We assume further that $g(0,0,0,0)=0$. This gives the following set of defining conditions for the considered singularity:

$$
f(0,0,0,0)=0, \quad \frac{\partial f}{\partial x}(0,0,0,0)=0, \quad g(0,0,0,0)=0
$$

with the nondegeneracy assumptions

$$
\frac{\partial^{2} f}{\partial x^{2}}(0,0,0,0) \neq 0, \quad \frac{\partial f}{\partial y}(0,0,0,0) \neq 0 .
$$

This implies that the critical manifold has a nondegenerate fold point for $\lambda$ in a suitable interval. Without loss of generality we assume that the fold point is $(0,0)$ for all values of $\lambda$. This can always be achieved by a $\lambda$-dependent translation. The remaining nondegeneracy assumptions defining a canard point are

$$
\frac{\partial g}{\partial x}(0,0,0,0) \neq 0, \quad \frac{\partial g}{\partial \lambda}(0,0,0,0) \neq 0 .
$$


These conditions insure that the nullcline $g(x, y, \lambda, 0)=0$ is transverse to the critical manifold $S$ and the intersection point of $S$ and $g(x, y, \lambda, 0)=0$ passes through the fold point $(0,0)$ with nonzero speed as $\lambda$ varies.

Let the critical manifold $S$, its left and right branches $S_{a}$ and $S_{r}$, and the neighborhoods $U$ and $V$ be defined as in section 2. The manifolds $S_{a, \varepsilon}$ and $S_{r, \varepsilon}$ exist outside of $V$ just as they did for a simple fold. Here we ask basically the same question, namely, How can $S_{a}$ and $S_{r}$ be extended? The situation is, however, quite different, since, for special choices of $\lambda$ and $\varepsilon, S_{a, \varepsilon}$ extends to $S_{r, \varepsilon}$. This is caused by the special structure of the slow flow for $\lambda=0$.

As in the case of the fold point, the reduced dynamics is governed by the equation

$$
\dot{x}=\frac{g(x, \varphi(x), 0,0)}{\varphi^{\prime}(x)},
$$

where $\varphi(x)$ is obtained by solving the equation $f(x, y, 0,0)$ for $y$ as a function of $x$. It follows from the above assumptions that the right-hand side of (3.5) is a smooth function at the origin. Let $x_{0}(t)$ denote a maximal solution of (3.5) with the property that $x_{0}(0)=0$. It follows that $x_{0}(t)$ exists and passes through the fold point (see Figure 3.1a). If $x_{0}(t)$ connects $S_{a}$ to $S_{r}$ then, heuristically, one can expect a connection from $S_{a, \varepsilon}$ to $S_{r, \varepsilon}$. In what follows we show that such a connection exists along a curve in the $(\lambda, \varepsilon)$-plane.

Remark 3.1. The case when $x_{0}(t)$ connects $S_{r}$ to $S_{a}$ can also be treated with the methods of this article, but it is less interesting and will be omitted.

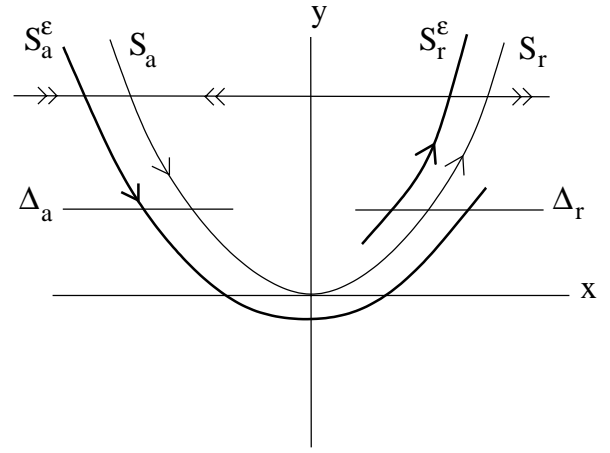

(a)

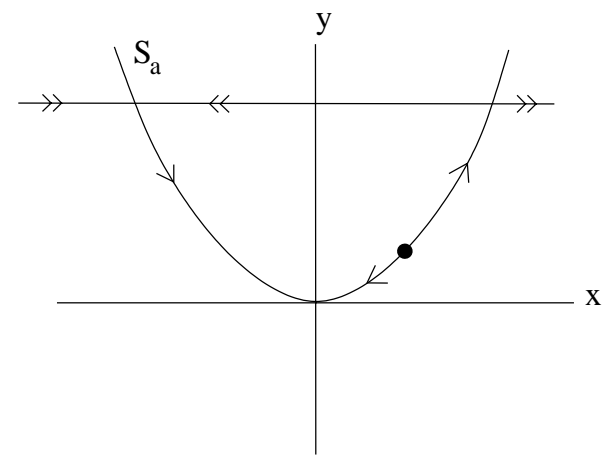

(b)

FIG. 3.1. Reduced flow. (a) $\lambda=0$, (b) $\lambda>0$.

It follows from assumptions (3.2) and (3.3) that using simple coordinate changes (scaling and translations) one can transform (3.1) to the canonical form

$$
\begin{aligned}
& x^{\prime}=-y h_{1}(x, y, \lambda, \varepsilon)+x^{2} h_{2}(x, y, \lambda, \varepsilon)+\varepsilon h_{3}(x, y, \lambda, \varepsilon), \\
& y^{\prime}=\varepsilon\left( \pm x h_{4}(x, y, \lambda, \varepsilon)-\lambda h_{5}(x, y, \lambda, \varepsilon)+y h_{6}(x, y, \lambda, \varepsilon)\right),
\end{aligned}
$$

where

$$
\begin{aligned}
& h_{3}(x, y, \lambda, \varepsilon)=O(x, y, \lambda, \varepsilon), \\
& h_{j}(x, y, \lambda, \varepsilon)=1+O(x, y, \lambda, \varepsilon), \quad j=1,2,4,5 .
\end{aligned}
$$

We assume that the sign in front of the term $x h_{4}$ is positive. In this case, $x_{0}(t)$ connects $S_{a}$ to $S_{r}$. Clearly, the signs of the various terms in the above equation 
correspond to a certain choice of signs in the nondegeneracy conditions made earlier. The parameter $\lambda$ has been rescaled such that the reduced flow has an equilibrium on $S_{r}$ for $\lambda>0$ (see Figure 3.1b).

We introduce the following notation:

$$
\begin{aligned}
& a_{1}=\frac{\partial h_{3}}{\partial x}(0,0,0,0), \quad a_{2}=\frac{\partial h_{1}}{\partial x}(0,0,0,0), \quad a_{3}=\frac{\partial h_{2}}{\partial x}(0,0,0,0), \\
& a_{4}=\frac{\partial h_{4}}{\partial x}(0,0,0,0), \quad a_{5}=h_{6}(0,0,0,0),
\end{aligned}
$$

and define

$$
A=-a_{2}+3 a_{3}-2 a_{4}-2 a_{5} .
$$

The constant $A$ will show up in various computations and results related to the analysis of the dynamics near the canard point. In particular, we will need the genericity condition $A \neq 0$ in the analysis of canard explosion in [14].

For $j=a, r$ let $\Delta_{j}=\left\{\left(x, \rho^{2}\right), x \in I_{j}\right\}$ be a section of $S_{j}$ near the fold point with $\rho$ sufficiently small and suitable intervals $I_{j}$ (see Figure 3.1a). Define $q_{j, \varepsilon}=\Delta_{j} \cap S_{j, \varepsilon}$. Let $\pi$ be the transition map for the flow of (3.1) from $\Delta_{a}$ to $\Delta_{r}$. The following theorem describes the behavior of $S_{a, \varepsilon}$ and $S_{r, \varepsilon}$ near the canard point.

THEOREM 3.1. Assume that system (3.1) satisfies the defining conditions (3.2)(3.4) of a canard point. Assume that the solution $x_{0}(t)$ of the reduced problem connects $S_{a}$ to $S_{r}$. Then there exists $\varepsilon_{0}>0$ and a smooth function $\lambda_{c}(\sqrt{\varepsilon})$ defined on $\left[0, \varepsilon_{0}\right]$ such that for $\varepsilon \in\left(0, \varepsilon_{0}\right]$ the following assertions hold:

1. $\pi\left(q_{a, \varepsilon}\right)=q_{r, \varepsilon}$ if and only if $\lambda=\lambda_{c}(\sqrt{\varepsilon})$.

2. The function $\lambda_{c}$ has the expansion

$$
\lambda_{c}(\sqrt{\varepsilon})=-\left(\frac{a_{1}+a_{5}}{2}+\frac{1}{8} A\right) \varepsilon+O\left(\varepsilon^{3 / 2}\right) .
$$

3. The transition map $\pi$ is defined only for $\lambda$ in an interval around $\lambda_{c}(\sqrt{\varepsilon})$ of width $O\left(e^{-c / \varepsilon}\right)$ for some $c>0$.

4 .

$$
\left.\frac{\partial}{\partial \lambda}\left(\pi\left(q_{a, \varepsilon}\right)-q_{r, \varepsilon}\right)\right|_{\lambda=\lambda_{c}(\sqrt{\varepsilon})}>0
$$

Remark 3.2. For $\lambda=\lambda_{c}(\sqrt{\varepsilon})$ the slow manifold $S_{a, \varepsilon}$ extends to the slow manifold $S_{r, \varepsilon}$, i.e., the slow manifold consists of a single canard solution. We use the term canard solution only for solutions with this property. In other works all solutions of system (1.1) which follow $S_{r, \varepsilon}$ for a time interval of order $O(1)$ are called canard solutions. The solution described in the above theorem is then called a maximal canard solution.

For the special case of the van der Pol equation the above results are contained in [6]. Here we treat the general case of a canard point and identify the important parameter $A$. Also, besides using the same blow-up our proof of the existence of a canard solution based on extending slow manifolds combined with a Melnikov-type argument is new. 
3.2. Blow-up. The analysis in this section is, in many aspects, similar to that in section 2. In particular we apply a blow-up transformation, yet the weights (powers of $r$ ) must be different than in the case of simple fold. Furthermore, the parameter $\lambda$ is now included in the blow-up. The blow-up transformation $\Phi$ maps $B=S^{2} \times$ $[-\mu, \mu] \times[0, \rho]$ to $\mathbb{R}^{4}$ according to

$$
x=\bar{r} \bar{x}, \quad y=\bar{r}^{2} \bar{y}, \quad \varepsilon=\bar{r}^{2} \bar{\varepsilon}, \quad \lambda=\bar{r} \bar{\lambda} .
$$

The constants $\mu$ and $\rho$ are chosen small enough such that equations (3.6) are valid in $\Phi(B)$. Let $\bar{X}$ denote the corresponding blown up vector field. In section 2 we used the charts $K_{1}, K_{2}$, and $K_{3}$ to obtain the dynamics of $\bar{X}$. Here charts $K_{1}$ and $K_{2}$ are sufficient to describe the relevant phenomena. In chart $K_{1}$, the blow-up transformation (3.9) is

$$
x=r_{1} x_{1}, \quad y=r_{1}^{2}, \quad \varepsilon=r_{1}^{2} \varepsilon_{1}, \quad \lambda=r_{1} \lambda_{1},
$$

where $\left(x_{1}, r_{1}, \varepsilon_{1}, \lambda_{1}\right)$ are the coordinates in $\mathbb{R}^{4}$. In chart $K_{2}$, the blow-up transformation $(3.9)$ is

$$
x=r_{2} x_{2}, \quad y=r_{2}^{2} y_{2}, \quad \varepsilon=r_{2}^{2}, \quad \lambda=r_{2} \lambda_{2},
$$

where $\left(x_{2}, y_{2}, r_{2}, \lambda_{2}\right)$ are the coordinates in $\mathbb{R}^{4}$. A simple computation gives the following lemma.

Lemma 3.2. Let $\kappa_{12}$ denote the change of coordinates from $K_{1}$ to $K_{2}$. Then $\kappa_{12}$ is given by

$$
x_{2}=x_{1} \varepsilon_{1}^{-1 / 2}, \quad y_{2}=\varepsilon_{1}^{-1}, \quad r_{2}=r_{1} \varepsilon_{1}^{1 / 2}, \quad \lambda_{2}=\varepsilon_{1}^{-1 / 2} \lambda_{1} \quad \text { for } \varepsilon_{1}>0 .
$$

Similarly, $\kappa_{21}=\kappa_{12}^{-1}$ is given by

$$
x_{1}=x_{2} y_{2}^{-1 / 2}, \quad r_{1}=r_{2} y_{2}^{1 / 2}, \quad \varepsilon_{1}=y_{2}^{-1}, \quad \lambda_{1}=\lambda_{2} y_{2}^{-1 / 2} \quad \text { for } y_{2}>0 .
$$

3.3. Dynamics in chart $\boldsymbol{K}_{\mathbf{2}}$ - preliminary analysis. After dividing out a factor $r_{2}$ in a manner analogous to that in section 2, the transformed equations (3.6) have the form

$$
\begin{aligned}
& x_{2}^{\prime}=-y_{2}+x_{2}^{2}+r_{2} G_{1}\left(x_{2}, y_{2}\right)+O\left(r_{2}\left(\lambda_{2}+r_{2}\right)\right), \\
& y_{2}^{\prime}=x_{2}-\lambda_{2}+r_{2} G_{2}\left(x_{2}, y_{2}\right)+O\left(r_{2}\left(\lambda_{2}+r_{2}\right)\right),
\end{aligned}
$$

where

$$
G\left(x_{2}, y_{2}\right)=\left(\begin{array}{c}
G_{1}\left(x_{2}, y_{2}\right) \\
G_{2}\left(x_{2}, y_{2}\right)
\end{array}\right)=\left(\begin{array}{c}
a_{1} x_{2}-a_{2} x_{2} y_{2}+a_{3} x_{2}^{3} \\
a_{4} x_{2}^{2}+a_{5} y_{2}
\end{array}\right)
$$

Remark 3.3. It turns out that for $r_{2}=\lambda_{2}=0$ the system (3.14) is integrable. For this reason the $O\left(r_{2}\right)$ and $O\left(\lambda_{2}\right)$ terms are crucial for the analysis.

Setting $r_{2}=\lambda_{2}=0$ in (3.14) we obtain

$$
\begin{aligned}
& x_{2}^{\prime}=-y_{2}+x_{2}^{2}, \\
& y_{2}^{\prime}=x_{2} .
\end{aligned}
$$

Equation (3.15) is integrable. More precisely, we have the following lemma. 
LEMma 3.3. The function

$$
H\left(x_{2}, y_{2}\right)=\frac{1}{2} e^{-2 y_{2}}\left(y_{2}-x_{2}^{2}+\frac{1}{2}\right)
$$

is a constant of motion for (3.15).

Proof. A computation gives

$$
\begin{aligned}
& x_{2}^{\prime}=e^{2 y_{2}} \frac{\partial H}{\partial y_{2}}\left(x_{2}, y_{2}\right), \\
& y_{2}^{\prime}=-e^{2 y_{2}} \frac{\partial H}{\partial x_{2}}\left(x_{2}, y_{2}\right) .
\end{aligned}
$$

The result follows.

Lemma 3.3 implies that the solutions of (3.15) are determined by the level curves of $H\left(x_{2}, y_{2}\right)$. Observe that (3.15) has an equilibrium at $(0,0)$ of center type, surrounded by a family of periodic orbits $H\left(x_{2}, y_{2}\right)=h, h \in(0,1 / 4)$. The sets $H\left(x_{2}, y_{2}\right)=$ $h, h \leq 0$, correspond to unbounded solutions. The locus of the solution determined by $h=0$ is the parabola $x_{2}^{2}-y_{2}=1 / 2$, and this solution is given by

$$
\gamma_{c, 2}\left(t_{2}\right)=\left(x_{c, 2}\left(t_{2}\right), y_{c, 2}\left(t_{2}\right)\right)=\left(\frac{1}{2} t_{2}, \frac{1}{4} t_{2}^{2}-\frac{1}{2}\right), \quad t_{2} \in \mathbb{R} .
$$

The special solution $\bar{\gamma}_{c}$ is of central importance to the canard phenomenon. We will see that $\bar{\gamma}_{c}$ connects the endpoint $p_{a}$ of the critical manifold $S_{a}$ across the sphere $S^{2}$ to the endpoint $p_{r}$ of the critical manifold $S_{r}$. As in the analysis of the fold point, the points $p_{a}$ and $p_{r}$ lie on the equator of $S^{2}$ and will be studied in chart $K_{1}$. Our goal is to investigate how this connection breaks under perturbation. The tool for this investigation will be a variant of the Melnikov method in which again both charts $K_{1}$ and $K_{2}$ will be used.

3.4. Dynamics in chart $\boldsymbol{K}_{\mathbf{1}}$. We proceed in a manner analogous to that in section 2.5 and obtain the following system of equations:

$$
\begin{aligned}
x_{1}^{\prime}= & -1+x_{1}^{2}+r_{1}\left(a_{1} \varepsilon_{1} x_{1}-a_{2} x_{1}+a_{3} x_{1}^{3}\right)-\frac{1}{2} \varepsilon_{1} x_{1} F\left(x_{1}, r_{1}, \varepsilon_{1}, \lambda_{1}\right) \\
& +O\left(r_{1}\left(r_{1}+\lambda_{1}\right)\right), \\
r_{1}^{\prime}= & \frac{1}{2} r_{1} \varepsilon_{1} F\left(x_{1}, r_{1}, \varepsilon_{1}, \lambda_{1}\right), \\
\varepsilon_{1}^{\prime}= & -\varepsilon_{1}^{2} F\left(x_{1}, r_{1}, \varepsilon_{1}, \lambda_{1}\right), \\
\lambda_{1}^{\prime}= & -\frac{1}{2} \lambda_{1} \varepsilon_{1} F\left(x_{1}, r_{1}, \varepsilon_{1}, \lambda_{1}\right),
\end{aligned}
$$

where

$$
F\left(x_{1}, r_{1}, \varepsilon_{1}, \lambda_{1}\right)=x_{1}-\lambda_{1}+r_{1}\left(a_{4} x_{1}^{2}+a_{5}\right)+O\left(r_{1}\left(r_{1}+\lambda_{1}\right)\right) .
$$

It suffices to consider $\lambda_{1} \in(-\mu, \mu)$, where $\mu>0$ can be chosen small. Many features of the dynamics in chart $K_{1}$ are analogous to section 2.5 ; therefore, we provide fewer details. The hyperplanes $r_{1}=0, \varepsilon_{1}=0$, and $\lambda_{1}=0$ are invariant, and the invariant line $l_{1}:=\left\{\left(x_{1}, 0,0,0\right): x_{1} \in \mathbb{R}\right\}$ contains two equilibria $p_{a}=(-1,0,0,0)$ and $p_{r}=$ $(1,0,0,0)$ which are endpoints of lines of equilibria $S_{a, 1}$ and $S_{r, 1}$, respectively. For the 
flow on the line $l_{1}$, the equilibrium $p_{a}$ is attracting and $p_{r}$ is repelling. Considered as equilibria of system (3.18), both equilibria have a triple eigenvalue zero.

In the invariant plane $r_{1}=\lambda_{1}=0$ system (3.18) reduces to

$$
\begin{aligned}
& x_{1}^{\prime}=-1+x_{1}^{2}-\frac{1}{2} \varepsilon_{1} x_{1}^{2}, \\
& \varepsilon_{1}^{\prime}=-\varepsilon_{1}^{2} x_{1} .
\end{aligned}
$$

Consequently, the sign of $\varepsilon_{1}^{\prime}$ is negative for initial conditions near $p_{r}$, which implies that the repelling center manifold $N_{r, 1}$ at $p_{r}$ in the half space $\varepsilon_{1}>0$ is unique. The attracting center manifold $N_{a, 1}$ at $p_{a}$ in the half space $\varepsilon_{1}>0$ is also unique, just as in section 2.5. Let

$$
D_{1}:=\left\{\left(x_{1}, r_{1}, \varepsilon_{1}, \lambda_{1}\right):-2<x_{1}<2,0 \leq r_{1} \leq \rho, 0 \leq \varepsilon_{1} \leq \delta,-\mu<\lambda_{1}<\mu\right\}
$$

where $\delta, \rho$, and $\mu$ will be chosen small.

Proposition 3.4. Choose $c_{1}<2<c_{2}$. The constants $\rho, \delta$ and $\mu$ can be chosen sufficiently small such that the following assertions hold for system (3.18):

1. There exists an attracting three-dimensional $C^{k}$-center manifold $M_{a, 1}$ at $p_{a}$ that contains the line of equilibria $S_{a, 1}$ and the center manifold $N_{a, 1}$. In $D_{1}$ the manifold $M_{a, 1}$ is given as a graph $x_{1}=h_{a}\left(r_{1}, \varepsilon_{1}, \lambda_{1}\right)$. The branch of $N_{a, 1}$ in $r_{1}=\lambda_{1}=0, \varepsilon_{1}>0$ is unique and equal to $\gamma_{c, 1}:=\kappa_{21}\left(\gamma_{c, 2}\right)$, where $\gamma_{c, 2}$ is the part of the special trajectory introduced in section 3.3, corresponding to $x_{2}$ close to $-\infty$.

2. There exists a repelling three-dimensional $C^{k}$-center manifold $M_{r, 1}$ at $p_{r}$ which contains the line of equilibria $S_{r, 1}$ and the center manifold $N_{r, 1}$. In $D_{1}$ the manifold $M_{r, 1}$ is given as a graph $x_{1}=h_{r}\left(r_{1}, \varepsilon_{1}, \lambda_{1}\right)$. The branch of $N_{r, 1}$ in $r_{1}=\lambda_{1}=0, \varepsilon_{1}>0$ is unique and equal to $\kappa_{21}\left(\gamma_{c, 2}\right)$ for $x_{2}$ close to $\infty$.

3. There exists a stable invariant foliation $\mathcal{F}^{s}$ with base $M_{a, 1}$ and one-dimensional fibers. There exist positive constants $K_{a, 1}$ and $K_{a, 2}$ such that the contraction along $\mathcal{F}^{s}$ in a time interval of length $T$ can be estimated by $K_{a, 2} e^{-c_{2} T}$ from below and by $K_{a, 1} e^{-c_{1} T}$ from above.

4. There exists an unstable invariant foliation $\mathcal{F}^{u}$ with base $M_{r, 1}$ and onedimensional fibers. There exist positive constants $K_{r, 1}$ and $K_{r, 2}$ such that the expansion along $\mathcal{F}^{u}$ in a time interval of length $T$ can be estimated by $K_{r, 1} e^{c_{1} T}$ from below and by $K_{r, 2} e^{c_{2} T}$ from above.

Proof. The proof is analogous to the proof of Proposition 3.4.

We now define the following sections:

$$
\begin{aligned}
& \Sigma_{a, 1}^{\text {in }}:=\left\{\left(x_{1}, r_{1}, \varepsilon_{1}, \lambda_{1}\right) \in D_{1}: r_{1}=\rho,\left|1+x_{1}\right|<\beta\right\}, \\
& \Sigma_{a, 1}^{\text {out }}:=\left\{\left(x_{1}, r_{1}, \varepsilon_{1}, \lambda_{1}\right) \in D_{1}: \varepsilon_{1}=\delta,\left|1+x_{1}\right|<\beta\right\}, \\
& \Sigma_{r, 1}^{\text {in }}:=\left\{\left(x_{1}, r_{1}, \varepsilon_{1}, \lambda_{1}\right) \in D_{1}: \varepsilon_{1}=\delta,\left|1-x_{1}\right|<\beta\right\}, \\
& \Sigma_{r, 1}^{\text {out }}:=\left\{\left(x_{1}, r_{1}, \varepsilon_{1}, \lambda_{1}\right) \in D_{1}: r_{1}=\rho,\left|1-x_{1}\right|<\beta\right\},
\end{aligned}
$$

where $\beta>0$ is chosen small. For $j=a, r$, let $\Pi_{j, 1}$ be the transition map defined by the flow of (3.18a) from section $\Sigma_{j, 1}^{i n}$ to $\sum_{j, 1}^{o u t}$. With these definitions, results analogous to Lemma 2.7 and Proposition 2.8 hold for the canard point as well. 
3.5. Phase portrait on $\boldsymbol{S}_{\mathbf{0}}^{2,+}$. Based on the analysis in charts $K_{1}$ and $K_{2}$, we can now describe the dynamics of $\bar{X}$ restricted to $S_{0}^{2,+}$, i.e., for $\bar{r}=\bar{\lambda}=0$. The equator $S^{1}$ is invariant. On $S^{1}$ there are four equilibria: $p_{a}, p_{r}, q_{\text {in }}, q_{\text {out }}$. These equilibria are hyperbolic for the flow on $S^{1}$, the points $p_{a}$ and $q_{\text {out }}$ are attracting, and $p_{r}$ and $q_{i n}$ are repelling. The special trajectory $\bar{\gamma}_{c}$ is a connecting orbit between $p_{a}$ and $p_{r}$. Besides $\bar{\gamma}_{c}$, there are three types of orbits in $S^{2,+}$ : a concentric family of periodic orbits, an equilibrium of center type, and a family of orbits joining $q_{i n}$ to $q_{\text {out }}$. The corresponding phase portrait is shown in Figure 3.2.

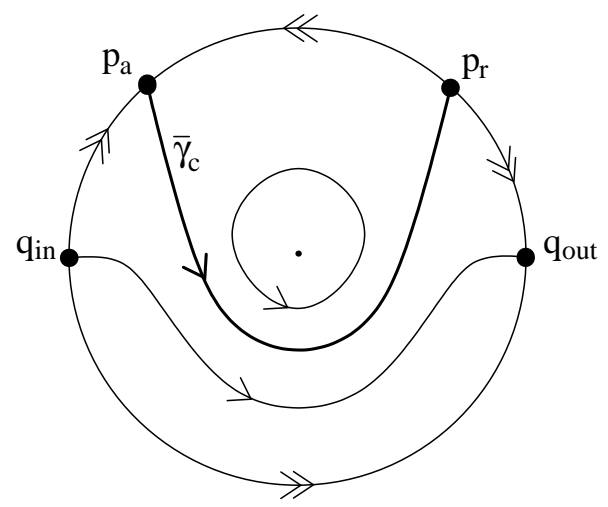

FIG. 3.2. Blow-up of a canard point restricted to $S_{0}^{2,+}$ for $\bar{\lambda}=0$.

Remark 3.4. It turns out that the connection $\bar{\gamma}_{c}$ from $p_{a}$ to $p_{r}$ breaks for $\bar{\lambda} \neq 0$. Some important aspects of the dynamics near $S_{0}^{2,+}$ leading to Theorem 3.1 can be understood by investigating whether the intersection of the three-dimensional center manifolds $\bar{M}_{a}$ and $\bar{M}_{r}$ at $\bar{\gamma}_{c}$ is transverse. The relevant computation is carried out in the next section. Bifurcations from the center equilibrium and the family of periodic orbits and their relevance to canard explosion are studied in [14].

3.6. Melnikov computation of the separation between $M_{a, 2}$ and $M_{r, 2}$. The analysis of the previous sections implies that $M_{a, 2}$ and $M_{r, 2}$ intersect along $\gamma_{c, 2}$ for $r_{2}=\lambda_{2}=0$. To prove Theorem 3.1 we compute the first order separation between $M_{a, 2}$ and $M_{r, 2}$ with respect to $r_{2}$ and $\lambda_{2}$. The splitting of the manifolds off the sphere for $\lambda_{2}=0$ is shown in Figure 3.3. All functions defined below are considered as functions of $r_{2} \in[0, \rho]$ and $\lambda_{2} \in(-\mu, \mu)$, for small $\rho>0$ and $\mu>0$, without indicating this dependence explicitly. In the formulas below we drop the subscript of the time variable $t_{2}$ from chart $K_{2}$, i.e., $t=t_{2}$.

Let $\gamma_{a, 1}$ be the trajectory of system (3.18) contained in $M_{a, 1}$ for which $r_{1} \sqrt{\varepsilon_{1}}=r_{2}$. Let $\gamma_{a, 2}(t)=\left(x_{a, 2}(t), y_{a, 2}(t)\right)$ be the continuation of $\gamma_{a, 1}$ to chart $K_{2}$, i.e., $\gamma_{a, 2}$ is a solution of (3.14), parametrized such that $x_{a, 2}(0)=0$. Analogously, let $\gamma_{r, 1}$ be the trajectory of (3.18) contained in $M_{r, 1}$ for which $r_{1} \sqrt{\varepsilon_{1}}=r_{2}$ and let $\gamma_{r, 2}(t)=\left(x_{r, 2}(t), y_{r, 2}(t)\right)$ be the corresponding, backward continued solution of (3.14) parametrized such that $x_{r, 2}(0)=0$.

With these definitions, measuring the separation of $M_{a, 2}$ and $M_{r, 2}$ corresponds to measuring $y_{a, 2}(0)-y_{r, 2}(0)$, which is equivalent to estimating the distance function

$$
\mathcal{D}_{c}\left(r_{2}, \lambda_{2}\right):=H\left(0, y_{a, 2}(0)\right)-H\left(0, y_{r, 2}(0)\right)
$$




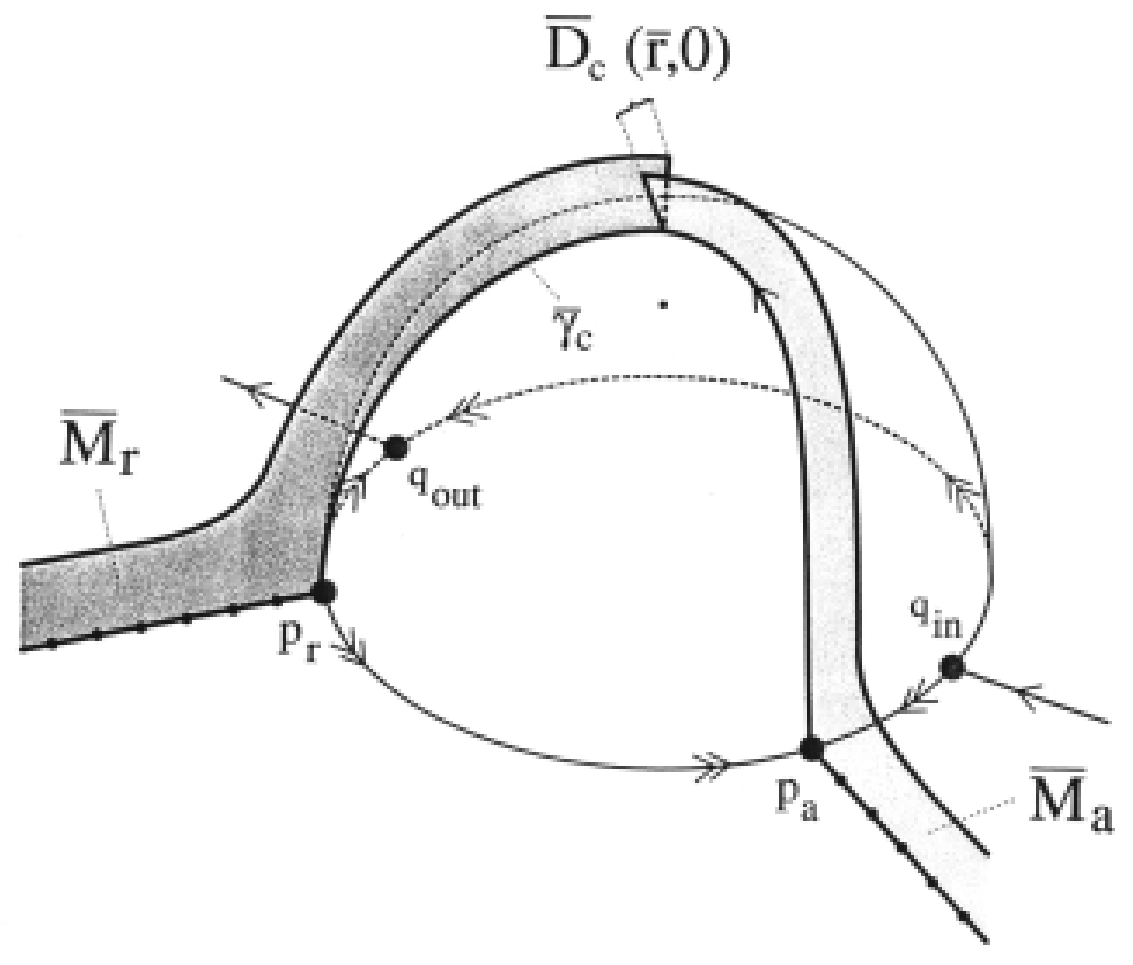

FIG. 3.3. Splitting of $\bar{M}_{a}$ and $\bar{M}_{r}$ for $\bar{\lambda}=0$.

since $\frac{\partial H}{\partial y_{2}}\left(0, y_{2}\right) \neq 0$ for $y_{2}<0$. We define

$$
d_{r_{2}}=\int_{-\infty}^{\infty} \operatorname{grad} H\left(\gamma_{c, 2}(t)\right) \cdot G\left(\gamma_{c, 2}(t)\right) d t
$$

where $G$ is the function defined in section 3.3. Similarly we define

$$
d_{\lambda_{2}}=\int_{-\infty}^{\infty} \operatorname{grad} H\left(\gamma_{c, 2}(t)\right) \cdot\left(\begin{array}{c}
0 \\
-1
\end{array}\right) d t
$$

We will prove the following result.

Proposition 3.5. For $\rho$ and $\mu$ small enough, the distance function $\mathcal{D}_{c}\left(r_{2}, \lambda_{2}\right)$ is a $C^{k}$-function and has the expansion

$$
\mathcal{D}_{c}\left(r_{2}, \lambda_{2}\right)=d_{r_{2}} r_{2}+d_{\lambda_{2}} \lambda_{2}+O(2) \text {. }
$$

Proof. By construction $\mathcal{D}_{c}$ is $C^{k}$ smooth and $\mathcal{D}_{c}(0,0)=0$. Thus we have to verify the expansion. We carry out the computation for $r_{2}$. The result for $\lambda_{2}$ can be obtained in a similar way. We set $\lambda_{2}=0$ and consider $r_{2} \in[0, \rho]$. We will show that

$$
H\left(0, y_{a, 2}(0)\right)=r_{2} \int_{-\infty}^{0} \operatorname{grad} H\left(\gamma_{c, 2}(t)\right) \cdot G\left(\gamma_{c, 2}(t)\right) d t+O\left(r_{2}^{2}\right) .
$$

An analogous argument yields

$$
H\left(0, y_{r, 2}(0)\right)=-r_{2} \int_{0}^{\infty} \operatorname{grad} H\left(\gamma_{c, 2}(t)\right) \cdot G\left(\gamma_{c, 2}(t)\right) d t+O\left(r_{2}^{2}\right)
$$


which implies the proposition.

We define $T\left(r_{2}, \delta\right)<0$ such that $y_{a, 2}(T)=\delta^{-1}$, where $\delta$ is the constant from the definition of $\Sigma_{1, a}^{o u t}$. We write

$$
H\left(0, y_{a, 2}(0)\right)=H\left(x_{a, 2}(T), \delta^{-1}\right)+\int_{T}^{0} \frac{d H}{d t}\left(\gamma_{a, 2}(t)\right) d t .
$$

By standard methods [4]

$$
\int_{T}^{0} \frac{d H}{d t}\left(\gamma_{a, 2}(t)\right) d t=r_{2} \int_{T}^{0} \operatorname{grad} H\left(\gamma_{a, 2}(t)\right) \cdot G\left(\gamma_{a, 2}(t)\right) d t+O\left(r_{2}^{2}\right) .
$$

It turns out to be very natural to compute $H\left(x_{a, 2}(T), \delta^{-1}\right)$ in chart $K_{1}$. We begin by parametrizing $\gamma_{a, 1}$ by $\varepsilon_{1}$, i.e.,

$$
\gamma_{a, 1}\left(\varepsilon_{1}\right)=\left(x_{a, 1}\left(\varepsilon_{1}\right), \frac{r_{2}}{\sqrt{\varepsilon_{1}}}, \varepsilon_{1}, 0\right), \quad \varepsilon_{1} \in\left[\left(\frac{r_{2}}{\rho}\right)^{2}, \delta\right],
$$

where $\rho$ is the constant used in the definition of $\Sigma_{a, 1}^{i n}$. Let $H_{1}=H \circ \kappa_{12}$, i.e.,

$$
H_{1}\left(x_{1}, \varepsilon_{1}\right)=H\left(\frac{x_{1}}{\sqrt{\varepsilon_{1}}}, \frac{1}{\varepsilon_{1}}\right)=e^{-2 / \varepsilon_{1}}\left(\frac{1}{4}+\frac{1}{2 \varepsilon_{1}}-\frac{x_{1}^{2}}{2 \varepsilon_{1}}\right) .
$$

We wish to estimate $H_{1}\left(x_{a, 1}(\delta), \delta\right)$. Note that $H_{1}\left(x_{a, 1}\left(\left(\frac{r_{2}}{\rho}\right)^{2}\right),\left(\frac{r_{2}}{\rho}\right)^{2}\right)$ is exponentially small in $r_{2}$. Hence,

$$
H_{1}\left(x_{a, 1}(\delta), \delta\right)=\int_{\left(\frac{r_{2}}{\rho}\right)^{2}}^{\delta} \frac{d}{d \varepsilon_{1}} H_{1}\left(x_{a, 1}\left(\varepsilon_{1}\right), \varepsilon_{1}\right) d \varepsilon_{1}+O\left(r_{2}^{2}\right) .
$$

From (3.26) we obtain

$$
\begin{aligned}
& \frac{\partial H_{1}}{\partial x_{1}}=-e^{-2 / \varepsilon_{1}} \frac{x_{1}}{\varepsilon_{1}}, \\
& \frac{\partial H_{1}}{\partial \varepsilon_{1}}=e^{-2 / \varepsilon_{1}} \frac{1}{\varepsilon_{1}^{2}}\left(\frac{1}{2} x_{1}^{2}-\frac{x_{1}^{2}}{\varepsilon_{1}}+\frac{1}{\varepsilon_{1}}\right) .
\end{aligned}
$$

Note that $\frac{d H_{1}}{d \varepsilon_{1}}$ evaluated along a trajectory of (3.18) is given by

$$
\frac{d H_{1}}{d \varepsilon_{1}}=\frac{\partial H_{1}}{\partial x_{1}} \frac{x_{1}^{\prime}}{\varepsilon_{1}^{\prime}}+\frac{\partial H_{1}}{\partial \varepsilon_{1}}
$$

where $x_{1}^{\prime}$ and $\varepsilon_{1}^{\prime}$ are given by (3.18a) and (3.18c), respectively. By using (3.27), expanding and using the relation $r_{1}=r_{2} / \sqrt{\varepsilon_{1}}$ to eliminate $r_{1}$, we obtain the following formula for $\frac{d H_{1}}{d \varepsilon_{1}}$ evaluated along a trajectory of (3.18):

$$
\frac{d H_{1}}{d \varepsilon_{1}}\left(x_{1}, \varepsilon_{1}\right)=e^{-2 / \varepsilon_{1}} \varepsilon_{1}^{-7 / 2}\left[r_{2}\left(a_{1} x_{1} \varepsilon_{1}-a_{2} x_{1}+a_{3} x_{1}^{3}+\frac{1-x_{1}^{2}}{x_{1}}\left(a_{4} x_{1}^{2}+a_{5}\right)\right)+\frac{1}{\sqrt{\varepsilon_{1}}} O\left(r_{2}^{2}\right)\right] .
$$

We set

$$
\eta\left(x_{1}, \varepsilon_{1}\right)=e^{-2 / \varepsilon_{1}} \varepsilon_{1}^{-7 / 2}\left(a_{1} x_{1} \varepsilon_{1}-a_{2} x_{1}+a_{3} x_{1}^{3}+\frac{1-x_{1}^{2}}{x_{1}}\left(a_{4} x_{1}^{2}+a_{5}\right)\right) .
$$


It follows that

$$
\int_{\left(\frac{r_{2}}{\rho}\right)^{2}}^{\delta} \frac{d}{d \varepsilon_{1}} H_{1}\left(x_{a, 1}\left(\varepsilon_{1}\right), \varepsilon_{1}\right) d \varepsilon_{1}=r_{2} \int_{\left(\frac{r_{2}}{\rho}\right)^{2}}^{\delta} \eta\left(x_{a, 1}\left(\varepsilon_{1}\right), \varepsilon_{1}\right) d \varepsilon_{1}+O\left(r_{2}^{2}\right),
$$

where we have used that the integral of the error term in (3.28) is $O\left(r_{2}^{2}\right)$ because of the exponentially small prefactor. To complete the computation, recall that $\left(x_{c, 1}\left(\varepsilon_{1}\right), \varepsilon_{1}\right)$ parametrizes $N_{a, 1}$, and, by center manifold theory,

$$
\left|x_{c, 1}\left(\varepsilon_{1}\right)-x_{a, 1}\left(\varepsilon_{1}\right)\right|=O\left(r_{1}\right)=O\left(\frac{r_{2}}{\sqrt{\varepsilon_{1}}}\right) .
$$

By using this estimate it follows that

$$
H_{1}\left(x_{a, 1}(\delta), \delta\right)=r_{2} \int_{0}^{\delta} \eta\left(x_{c, 1}\left(\varepsilon_{1}\right), \varepsilon_{1}\right) d \varepsilon_{1}+O\left(r_{2}^{2}\right),
$$

where we have again used the exponentially small prefactor of the integrand to estimate the error caused by first replacing $x_{a, 1}$ by $x_{c, 1}$ and then changing the interval of integration to $[0, \delta]$. By applying the change of variables formula, we transform this integral to chart $K_{2}$ and obtain

$$
\int_{0}^{\delta} \eta\left(x_{c, 1}\left(\varepsilon_{1}\right), \varepsilon_{1}\right) d \varepsilon_{1}=\int_{-\infty}^{T} \operatorname{grad} H\left(\gamma_{c, 2}(t)\right) \cdot G\left(\gamma_{c, 2}(t)\right) d t .
$$

The result follows.

Remark 3.5. The formulas for the constants $d_{r_{2}}$ and $d_{\lambda_{2}}$ are the usual Melnikov integrals for the splitting of saddle-saddle connections for perturbations of planar Hamiltonian vector fields. However, the situation considered above is not covered by the usual Melnikov theory.

Proof of Theorem 3.1. It follows from the above results that for $\left(r_{2}, \lambda_{2}\right) \in[0, \rho) \times$ $(-\mu, \mu)$ a connection from $S_{a, \varepsilon}$ to $S_{r, \varepsilon}$ exists if and only if

$$
\mathcal{D}_{c}\left(r_{2}, \lambda_{2}\right)=0 \text {. }
$$

We have shown that $\mathcal{D}_{c}\left(r_{2}, \lambda_{2}\right)=d_{r_{2}} r_{2}+d_{\lambda_{2}} \lambda_{2}+O(2)$. Hence, (3.30) can be solved for $\lambda_{2}$ by the implicit function theorem provided that $d_{\lambda_{2}} \neq 0$. The solution has the expansion

$$
\lambda_{2}=-\frac{d_{r_{2}}}{d_{\lambda_{2}}} r_{2}+O\left(r_{2}^{2}\right) .
$$

By using the parametrization of $\gamma_{c, 2}$ and repeated integration by parts, we compute

$$
\begin{aligned}
d_{r_{2}} & =\int_{-\infty}^{\infty} e^{-2 y_{2}}\left(-a_{1} x_{2}^{2}+\left(a_{2}-a_{4}+a_{5}\right) x_{2}^{2} y_{2}+\left(a_{4}-a_{3}\right) x_{2}^{4}-a_{5} y_{2}^{2}\right) d t \\
& =-\frac{e}{4}\left(a_{1}+a_{5}+\frac{1}{4} A\right) \int_{-\infty}^{\infty} e^{-t^{2} / 2} d t
\end{aligned}
$$

and

$$
d_{\lambda_{2}}=-\int_{-\infty}^{\infty} \frac{1}{2} e^{-2 y_{2}} d t=-\frac{e}{2} \int_{-\infty}^{\infty} e^{-t^{2} / 2} d t
$$


This proves assertion (1). Property (2) follows by applying transformation (3.11) to the the expansion (3.31). Assertion (3) follows from the above combined with Proposition 3.4 because $M_{a, 2}$ must be exponentially close to $M_{r, 2}$ to reach the section $\Sigma_{r, 1}^{o u t}$. Finally, the inequality $d_{\lambda_{2}}<0$ implies that the intersection of the slow manifolds breaks as described in assertion (4).

Remark 3.6. It is known that the function $\lambda_{c}$ from Theorem 3.1 which describes the canard curve actually has a power series in $\varepsilon$; see [3], [7], [19]. It is interesting to observe that this fact can be explained by a symmetry property of the blowup transformation. The form of the blow-up transformation (3.9) implies that the transformation

$$
(\bar{x}, \bar{y}, \bar{r}, \bar{\lambda}) \mapsto(-\bar{x}, \bar{y},-\bar{r},-\bar{\lambda})
$$

is a time-reversal symmetry of the blown-up vector field $\bar{X}$; i.e., it maps orbits to orbits. Note that for prescribed slow manifolds outside $V$, the canard curve is uniquely determined. Since the transformation (3.34) maps a canard curve to a canard curve, it follows that $-\lambda_{c, 2}\left(r_{2}\right)=\lambda_{c, 2}\left(-r_{2}\right)$ and consequently $\lambda_{c}\left(r_{2}\right)=\lambda_{c, 2}\left(-r_{2}\right)$. From this we conclude that an asymptotic expansion of the canard curve is quadratic in $r_{2}$ and hence is a power series in $\varepsilon$. If the original vector field is smooth and the slow manifolds are chosen smooth, then, by a theorem of Schwartz [21], $\lambda_{c}$ is a smooth function of $\varepsilon$.

The function $\lambda_{c}$ depends on the choice of the slow manifolds. However all slow manifolds are exponentially close and the corresponding functions $\lambda_{c}$ differ only by exponentially small terms and have the same asymptotic expansion.

Acknowledgments. The authors would like to thank Freddy Dumortier, Stephan van Gils, Alexandra Milik, and Martin Wechselberger for helpful discussions.

The authors also acknowledge the support and hospitality of the Institute for Mathematics and Its Applications at the University of Minnesota, where a part of this research was carried out.

\section{REFERENCES}

[1] V. I. Arnold, Ed., Dynamical Systems V, Bifurcation theory and catastrophe theory, Encyclopaedia Math. Sci. 5, Springer-Verlag, Berlin, 1989.

[2] E. Benoit, ED., Dynamic Bifurcations, Lecture Notes in Math. 1493, Springer, New York, 1991.

[3] E. Benoit, J. L. Callot, F. Diener, And M. Diener, Chasse au canards, Collect. Math., 31 (1981), pp. 37-119.

[4] S-N. Chow, C. Li, And D. Wang, Normal Forms and Bifurcation of Planar Vector Fields, Cambridge University Press, Cambridge, 1994.

[5] F. Dumortier, Techniques in the theory of local bifurcations: Blow-up, normal forms, nilpotent bifurcations, singular perturbations, in Bifurcations and Periodic Orbits of Vector Fields, D. Szlomiuk, ed., NATO Adv. Sci. Inst. Ser. C Math. Phys. Sci. 408, Kluwer, Dordrecht, the Netherlands, 1993, pp. 19-73.

[6] F. Dumortier and R. Roussarie, Canard cycles and center manifolds, Mem. Amer. Math. Soc. 577, Providence, 1996.

[7] W. Eckhaus, Relaxation oscillations including a standard chase on French ducks, in Asymptotic Analysis II, Lecture Notes in Math. 985, Springer, New York, 1983, pp. 449-494.

[8] N. Fenichel, Geometric singular perturbation theory, J. Differential Equations, 31 (1979), pp. 53-98.

[9] J. Grasman, Asymptotic Methods for Relaxation Oscillations and Applications, Springer, New York, 1987.

[10] J. Guckenheimer and P. Holmes, Nonlinear Oscillations, Dynamical Systems, and Bifurcations of Vector Fields, Springer, New York, 1983.

[11] C.K. R. T. Jones, Geometric singular perturbation theory, in Dynamical Systems, Lecture Notes in Math. 1609, Springer, New York, 1995, pp. 44-120. 
[12] J. Kevorkian And J. D. Cole, Perturbation Methods in Applied Mathematics, Springer, New York, 1981.

[13] M. Krupa And P. Szmolyan, Geometric analysis of the singularly perturbed planar fold, Multiple time scale dynamical systems, IMA Vol. Math. Appl., 122 (2000), pp. 89-116.

[14] M. Krupa And P. Szmolyan, Relaxation oscillation and canard explosion, J. Differential Equations, to appear, 2001.

[15] M. Krupa and P. Szmolyan, Transcritical and pitchfork singularities of critical manifolds, Nonlinearity, submitted, 2000.

[16] S.A. van Gils, M. Krupa, And P. Szmolyan, Asymptotic expansions using blow-up, Z. Angew. Math. Phys., submitted, 2000.

[17] A. Milik And P. Szmolyan, Multiple time scales and canards in a chemical oscillator, Multiple time scale dynamical systems, IMA Vol. Math. Appl., 122 (2000), pp. 117-140.

[18] E. F. Mishchenko And N. Kh. Rozov, Differential Equations with Small Parameters and Relaxation Oscillations, Plenum Press, New York, 1980.

[19] E. F. Mishchenko, Yu. S. Kolesov, A. Yu. Kolesov, and N. Kh. Rozov, Asymptotic Methods in Singularly Perturbed Systems, Monogr. Contemp. Math., Consultants Bureau, New York, 1994.

[20] L.S. Pontryagin, Asymptotic behavior of solutions of systems of differential equations with a small parameter in derivatives of highest order, Izv. Akad. Nauk. SSSR Ser. Mat., 21 (1957), pp. 605-626.

[21] G. SchwarTz, Smooth functions invariant under the action of a compact Lie group, Topology, 14 (1975), pp. 63-68.

[22] S. SternberG, On the nature of local homeomorphisms of Euclidean n-space II, Amer. J. Math., 80 (1958), pp. 623-631.

[23] M. Wechselberger, Singularly Perturbed Folds and Canards in $\mathbb{R}^{3}$, Ph.D. Thesis, TU-Wien, Wien, Austria, 1998. 